Louisiana State University

LSU Digital Commons

Faculty Publications

Department of Physics \& Astronomy

4-20-2012

\title{
Thirty years of SN 1980K: Evidence for light echoes
}

\author{
Ben E.K. Sugerman \\ Goucher College \\ Jennifer E. Andrews \\ University of Massachusetts Amherst \\ Michael J. Barlow \\ University College London \\ Geoffrey C. Clayton \\ Louisiana State University \\ Barbara Ercolano \\ Ludwig-Maximilians-Universität München
}

See next page for additional authors

Follow this and additional works at: https://digitalcommons.Isu.edu/physics_astronomy_pubs

\section{Recommended Citation}

Sugerman, B., Andrews, J., Barlow, M., Clayton, G., Ercolano, B., Ghavamian, P., Kennicutt, R., Krause, O., Meixner, M., \& Otsuka, M. (2012). Thirty years of SN 1980K: Evidence for light echoes. Astrophysical Journal, 749 (2) https://doi.org/10.1088/0004-637X/749/2/170

This Article is brought to you for free and open access by the Department of Physics \& Astronomy at LSU Digital Commons. It has been accepted for inclusion in Faculty Publications by an authorized administrator of LSU Digital Commons. For more information, please contact ir@lsu.edu. 


\section{Authors}

Ben E.K. Sugerman, Jennifer E. Andrews, Michael J. Barlow, Geoffrey C. Clayton, Barbara Ercolano, Parviz Ghavamian, Robert C. Kennicutt, Oliver Krause, Margaret Meixner, and Masaaki Otsuka 


\title{
THIRTY YEARS OF SN 1980K: EVIDENCE FOR LIGHT ECHOES
}

\author{
Ben E. K. Sugerman ${ }^{1}$, Jennifer E. Andrews ${ }^{2}$, Michael J. Barlow ${ }^{3}$, Geoffrey C. Clayton ${ }^{4}$, Barbara Ercolano ${ }^{5}$, \\ Parviz Ghavamian $^{6}$, Robert C. Kennicutt, Jr. $^{7}$, Oliver Krause ${ }^{8}$, Margaret MeiXner $^{9}$, and MasaAki Otsuka ${ }^{10}$ \\ ${ }^{1}$ Department of Physics and Astronomy, Goucher College, 1021 Dulaney Valley Road, Baltimore, MD 21208, USA; ben.sugerman@goucher.edu \\ 2 Department of Astronomy, University of Massachusetts, 710 North Pleasant Street, Amherst, MA 01003, USA \\ ${ }_{3}^{3}$ Department of Physics and Astronomy, University College London, Gower Street, London WC1E 6BT, UK \\ ${ }^{4}$ Department of Physics and Astronomy, Lousiana State University, 202 Nicholson Hall, Baton Roughe, LA 70803, USA \\ ${ }_{5}^{5}$ Excellence Cluster “Universe,” Universitäts-Sternwarte München, Scheinerstr. 1, 81679 München, Germany \\ ${ }^{6}$ Department of Physics, Astronomy and Geosciences, Towson University, Smith Hall, Towson, MD 21252, USA \\ ${ }^{7}$ Institute of Astronomy, University of Cambridge, Madingley Road, Cambridge, CM3 OHA, UK \\ ${ }^{8}$ Max Planck Institute for Astronomy, Königstuhl 17, 69117 Heidelberg, Germany \\ ${ }^{9}$ Space Telescope Science Institute, 3700 San Martin Drive, Baltimore, MD 21218, USA \\ ${ }^{10}$ Institute of Astronomy and Astrophysics, Academia Sinica, Taipei 10617, Taiwan, Republic of China \\ Received 2011 December 22; accepted 2012 February 14; published 2012 April 5
}

\begin{abstract}
We report optical and mid-infrared photometry of SN 1980K between 2004 and 2010, which shows slow monotonic fading consistent with previous spectroscopic and photometric observations made 8-17 yr after outburst. The slow rate of change over two decades suggests that this evolution may result from scattered and thermal light echoes off of extended circumstellar material. We present a semi-analytic dust radiative-transfer model that uses an empirically corrected effective optical depth to provide a fast and robust alternative to full Monte Carlo radiative-transfer modeling for homogenous dust at low to intermediate optical depths. We find that unresolved echoes from a thin circumstellar shell 14-15 lt-yr from the progenitor, and containing $\lesssim 0.02 M_{\odot}$ of carbon-rich dust, can explain the broadband spectral and temporal evolution. The size, mass, and dust composition are in good agreement with the contact discontinuity observed in scattered echoes around SN 1987A. The origin of slowly changing high-velocity [O I] and $\mathrm{H} \alpha$ lines is also considered. We propose an origin in shocked high-velocity metal-rich clumps of ejecta, rather than arising in the impact of ejecta on slowly moving circumstellar material, as is the case with hot spots in SN 1987A.
\end{abstract}

Key words: circumstellar matter - dust, extinction - methods: numerical - radiative transfer - supernovae: individual (SN 1980K)

\section{INTRODUCTION}

Nine supernovae ( $\mathrm{SNe}$ ) have been observed in the spiral galaxy NGC 6946 ( $d=5.9 \mathrm{Mpc}$; Karachentsev et al. 2000) in the past century, earning this nearby supernova factory the nickname the "Fireworks Galaxy" and making it a virtual laboratory for studying a variety of SN characteristics from progenitor identification through evolution into early remnant stages. With the Hubble and Spitzer Space Telescopes (HST and Spitzer, respectively) and large ground-based observatories all available during the past decade, a great deal of understandable interest has been paid to its recent SNe 2002hh, 2004et, and 2008S. Continued monitoring of its older population (SNe 1917A, 1939C, 1948B, 1968D, 1969P, and 1980K) provides an extraordinary opportunity to connect the early evolution $(\lesssim 10 \mathrm{yr})$ of young SNe with evolved remnants (e.g., Cas A, N103B). In this paper, we report and analyze optical and infrared (IR) data collected between 2004 and 2010 of SN 1980K.

First discovered on 1980 October 28 (Wild \& Barbon 1980), this core-collapse, linear (or type II-L) SN was recovered in $\mathrm{H} \alpha$ imaging in 1987 and spectroscopy in 1988 (Fesen \& Becker 1990), which revealed a faint continuum underlying a complex of high-velocity $\mathrm{H} \alpha$ and [OI] lines that had not been present during the first two years (Barbieri et al. 1982; Uomoto \& Kirshner 1986). Continued observations (Uomoto 1991; Fesen \& Matonick 1994; Fesen et al. 1995, 1999) have shown the total fluxes to have declined by about $25 \%$ over more than a decade, with the centers and fluxes of the individual highvelocity components changing on timescales of a few years. A detailed consideration of possible mechanisms led Fesen et al. (1999) to conclude that these lines originate in shocks driven by the SN blast into preexisting circumstellar material (CSM). The presence of such material has been inferred both from IR excesses observed up to $1 \mathrm{yr}$ after outburst (Dwek et al. 1983), which Dwek (1983) argued arose from thermal echoes off of nearby CSM, and from 6 and $20 \mathrm{~cm}$ radio evolution (Weiler et al. 1992), consistent with the forward blast impacting dense CSM laid down by the progenitor red supergiant a few tens of thousands of years prior to core collapse.

The presence of a faint continuum within all late-time spectra and the slow monotonic decrease in flux in all optical wavebands can also arise from a scattered-light echo (Couderc 1939) illuminating a large CSM shell or interstellar dust sheet. Whether from scattered or thermally reprocessed SN light, an echo arises when dust redirects SN light back into the line of sight, which arrives at the observer later than the original signal by the light-travel time along the longer path length. Indeed, a scattered echo has been considered for SN $1980 \mathrm{~K}$ by Chevalier (1986), who concluded that the flux evolution through the first $\sim 1 \mathrm{yr}$ was consistent with a scattering-echo model, but more observations were necessary to distinguish it from radioactiveenergy deposition.

In this paper, we present optical photometry of SN 1980K made by the HST and Gemini North and mid-IR photometry made by Spitzer, all between 2004 and 2010, as well as upper limits in the far-IR from Herschel Space Observatory imaging made in 2010 (Section 2). Combined with previously reported optical photometry, we show that the past decade of evolution is 
consistent with a slow, monotonic fading across all wavebands (Section 3 ) that can be explained by scattered and thermal echoes from a large $(r \sim 14$ lt-yr) shell of circumstellar dust. The basic behavior and modeling of light echoes are discussed in Section 4, followed by an exploration of the parameter space of possible shell properties, as well as a new, semi-analytic dust radiative-transfer model, in the Appendix. The consistency of the proposed light echoes with previous analyses of the highvelocity emission-line structures is discussed in Section 5.

\section{OBSERVATIONS}

\subsection{Imaging}

In the mid-infrared, SN $1980 \mathrm{~K}$ has been imaged at least yearly between 2004 June and 2008 July with Spitzer in the same field as SN 2004et with both the InfraRed Array Camera (IRAC) and Multiband Imaging Photometer for Spitzer (MIPS) as part of the original cold mission (GO-159, 20256, 20320, 30494, 40010), and continuing through 2010 with IRAC as part of its ongoing warm mission (GO-60071, 70008), often as part of the ongoing work of the SEEDS collaboration (Search for Evolution of Emission from Dust in Supernovae; PI: M. Barlow). Pipelinecalibrated imaging data were retrieved from the Spitzer archive and processed with mopex (Makovoz \& Khan 2005) to achieve enhanced resolutions of 0.75 pixel $^{-1}$ for IRAC and $1^{\prime \prime} .5$ pixel $^{-1}$ for MIPS.

SN 1980K was imaged once on chip 2 of the HST Wide Field and Planetary Camera 2 (WFPC2) in the F606W and F814W filters on 2008 January 19 (GO-11229). Pipeline-reduced data were combined using multidrizzle (Koekemoer et al. 2002) to achieve a final pixel scale of 0.1 pixel $^{-1}$. Two epochs of optical observations were also made with Gemini North in $g^{\prime} r^{\prime} i$ filters on 2005 August 5 and 2006 July 17 using the Gemini Multi-Object Spectrograph in imaging mode. Images were reduced and stacked using the IRAF gemini package, and instrumental counts were calibrated to Johnson-Cousins $V R_{c} I_{c}$ magnitudes using transformations from Welch et al. (2007).

NGC 6946 was imaged in the far-IR by Herschel in all wavebands on 2010 March 10 as part of the KINGFISH program (R. Kennicutt, P.I.). One arcmin fields surrounding the position of SN 1980K were extracted from the enhanced Photoconductor Array Camera and Spectrometer (PACS) and Spectral and Photometric Imaging REceiver (SPIRE) data products as explained in Kennicutt et al. (2011). Images of the field at representative wavelengths are shown in Figure 1.

\subsection{Archival Spectra}

Optical spectra of various wavelength ranges and resolution have been published for SN 1980K from maximum light in 1980 through late 1997 (Barbon et al. 1982; Uomoto \& Kirshner 1986; Leibundgut et al. 1991; Uomoto 1991; Fesen \& Becker 1990; Fesen \& Matonick 1994; Fesen et al. 1995, 1999). For this work, optical spectra from the first $\sim 100$ days (Barbon et al. 1982) that were digitized and flux calibrated by Benetti (1989) were acquired from the Asiago Supernova Archive. Spectra from Uomoto \& Kirshner (1986), Fesen \& Becker (1990), and Fesen et al. (1999) were extracted using DEXTER ${ }^{11}$ from fully printable scans or electronic articles archived in the Astrophysical Data System. ${ }^{12}$ The late-time spectrum from 1988 August (Fesen \& Becker 1990) was integrated over the Johnson $V$ and Cousins $R_{c}$

\footnotetext{
11 http://dc.sah.uni-heidelberg.de/sdexter

12 http://adsabs.harvard.edu
}

Table 1

Photometric Behavior of SN 1980K

\begin{tabular}{lccrccc}
\hline \hline $\begin{array}{l}\lambda_{0} \\
(\mu \mathrm{m})\end{array}$ & $\begin{array}{c}\text { Average Flux } \\
(\mu \mathrm{Jy})\end{array}$ & $\begin{array}{c}\text { Best-fit Slope } \\
\left(\mu \mathrm{Jy} \mathrm{yr}^{-1}\right)\end{array}$ & $N^{\mathrm{a}}$ & $Q^{\mathrm{b}}$ & $\begin{array}{c}F(\lambda) \text { at 23 yr } \\
(\mu \mathrm{Jy})\end{array}$ & $\begin{array}{c}F(\lambda) \text { at 28 yr } \\
(\mu \mathrm{Jy})\end{array}$ \\
\hline 0.55 & $1.7 \pm 0.4$ & $-0.044 \pm 0.039$ & 5 & 0.873 & $1.8 \pm 0.4$ & $1.6 \pm 0.4$ \\
0.64 & $3.1 \pm 0.7$ & $-0.043 \pm 0.057$ & 4 & 0.091 & $3.0 \pm 0.7$ & $2.8 \pm 0.7$ \\
0.80 & $1.9 \pm 0.5$ & $-0.006 \pm 0.100$ & 4 & 0.740 & $1.9 \pm 0.7$ & $1.9 \pm 0.7$ \\
3.6 & $1.8 \pm 0.5$ & $-0.067 \pm 0.103$ & 9 & 0.831 & $2.1 \pm 0.7$ & $1.8 \pm 0.7$ \\
4.5 & $5.9 \pm 0.6$ & $-0.352 \pm 0.090$ & 12 & 0.024 & $7.7 \pm 0.7$ & $5.9 \pm 0.8$ \\
5.8 & $16.8 \pm 5.2$ & $-1.936 \pm 1.769$ & 5 & 0.968 & $22 \pm 10$ & $13 \pm 10$ \\
8.0 & $51.9 \pm 13.4$ & $0.706 \pm 3.147$ & 11 & 0.552 & $50 \pm 21$ & $53 \pm 21$ \\
24. & $460 . \pm 28$. & $-14.73 \pm 8.15$ & 10 & 0.138 & $503 \pm 50$ & $430 \pm 50$ \\
70. & $<5900$. & $\ldots$ & 1 & $\cdots$ & $<5900$. & $<5900$. \\
100. & $<5900$. & $\ldots$ & 1 & $\ldots$ & $<5900$. & $<5900$. \\
160. & $<9900$. & $\ldots$ & 1 & $\ldots$ & $<9900$. & $<9900$. \\
250. & $<15000$. & $\ldots$ & 1 & $\ldots$ & $<15000$. & $<15000$. \\
\hline
\end{tabular}

Notes.

${ }^{a}$ Number of epochs in fit and average.

b The incomplete gamma-function $Q$ gives the goodness-of-fit probability.

filter response functions and the spectrum from 1997 November (Fesen et al. 1999) over $V, R_{c}$, and $I_{c}$, yielding estimates of $V=22.9$ and $R_{c}=22.1$ in 1988 and $V=23.3, R_{c}=22.4$, and $I_{c} \lesssim 22.9$ in 1997 . These are consistent with the broadband magnitudes $V=22.8 \pm 0.2, R=21.9 \pm 0.1$, and $I=22.2 \pm 0.3$ reported by Leibundgut et al. (1993) between 1990 and 1992.

\section{PHOTOMETRIC ANALYSIS}

\subsection{Astrometry}

Given that SN $1980 \mathrm{~K}$ is $\geqslant 25$ yr past maximum light in our data, it is important to verify the position of the point source identified as the SN. Fifteen stars in the HST/WFPC2 field were extracted from the USNO-B1 astrometric catalog (Monet et al. 2003) and compared to positions in the HST and Spitzer data to calibrate the world-coordinate systems using standard IRAF routines. These show that the point source at the center of panels (a)-(e) in Figure 1 is within $0{ }^{\prime} .1$ of the Very Large Array position of the SN as given in Montes et al. (1998). In particular, SN $1980 \mathrm{~K}$ is the right-hand point source at the very center of the HST image shown in Figure 1(a).

\subsection{Photometry}

In optical and mid-IR imaging, photometry was performed using the daophot point-spread function (PSF) fitting routines within IRAF, which also provide a robust estimate of uncertainty using a combination of Poisson noise, flat-fielding, and absolute calibration errors. The resulting fluxes and errors, as well as earlier data estimated from archival spectra, are shown in Figure 2. Also shown in gray are ranges of slopes that fit the data with standard least squares. The general trend is for a slow fading in time in almost all wavebands, although most data are consistent with the flux remaining approximately constant over the time period sampled. For this reason, the weightedaverage values for each filter are presented in Table 1 along with the least-squares slopes, and the averaged spectral energy distribution (SED) is plotted in Figure 3.

No point sources were detected at the position of the $\mathrm{SN}$ in Herschel data. Although there is a point-source-like feature at the correct position in the $160 \mu \mathrm{m}$ frame (Figure 1(h)), it has the same brightness as horizontal and vertical banding throughout the image, suggesting that it is coincident noise. To estimate the 


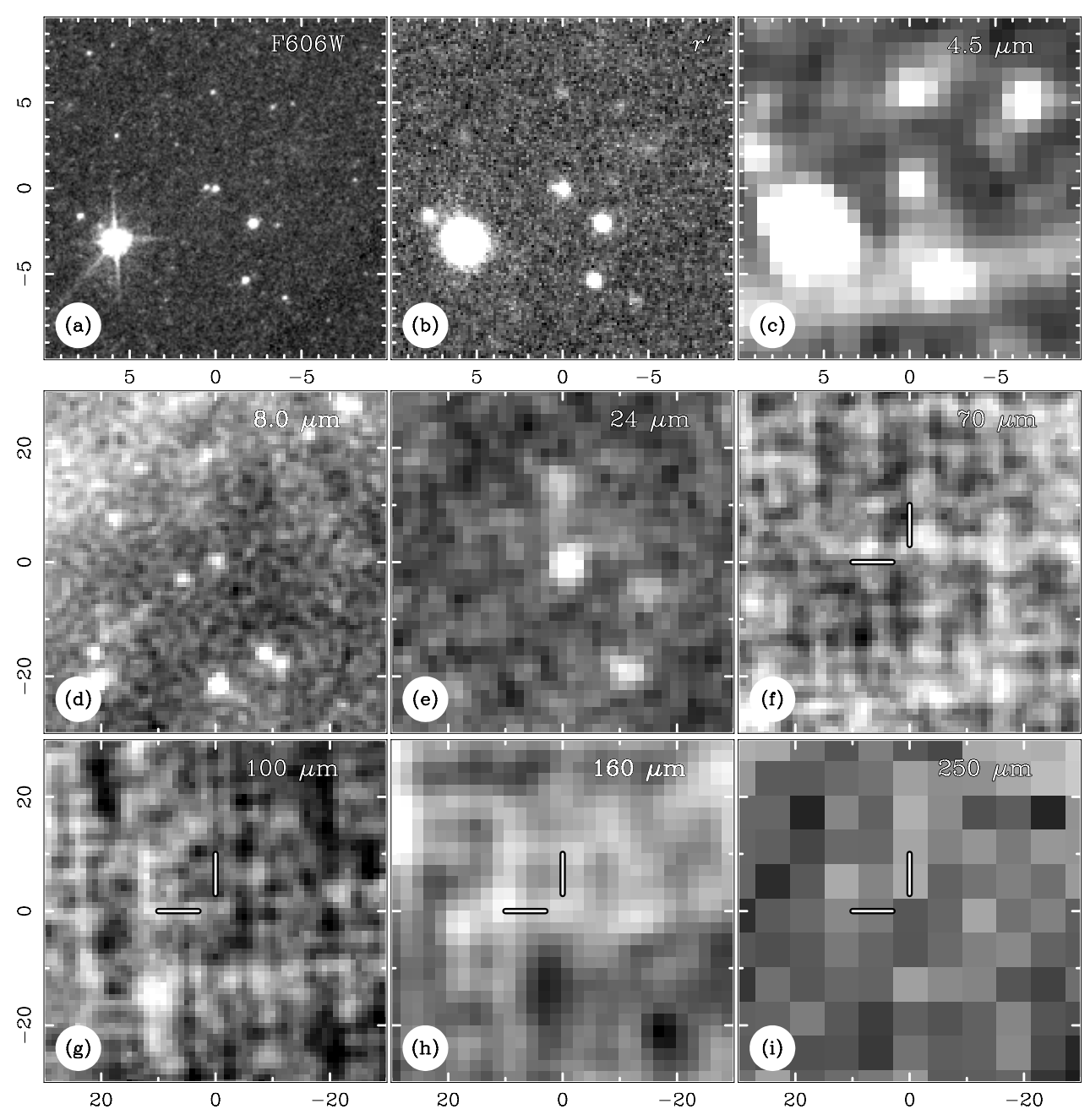

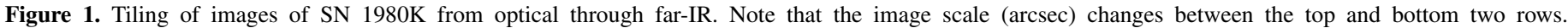

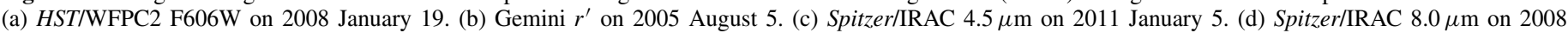
July 18. (e) Spitzer/MIPS $24 \mu \mathrm{m}$ on 2008 July 29. (f)-(i) Herschel/PACS and SPIRE on 2010 October 3 at $70 \mu \mathrm{m}, 100 \mu \mathrm{m}, 160 \mu \mathrm{m}$, and $250 \mu \mathrm{m}$, respectively.

upper limits in each frame, a grid of PSFs was added to each image with varying brightness until daophot could detect them. The resulting PSF fluxes are close to the upper limits quoted for each imager by the Herschel Science Centre ${ }^{13}$ and have been listed in Table 1 and plotted as downward arrows in Figure 3.

\subsection{Interpretation}

The mid-IR SED shown in Figure 3 is consistent with thermal emission from warm dust. Regardless of its origin, a zeroth-order estimate of the composition and mass of emitting particles can be made by assuming that all grains are visible (i.e., negligible internal extinction, which is reasonable for mid-IR wavelengths), have a single temperature $T$, and emit as blackbodies modified by their mass-absorption coefficients $\kappa_{\lambda}=\pi a^{2} Q_{\mathrm{abs}} / m_{g}$, where $a$ is the grain radius, $Q_{\mathrm{abs}}$ is the absorption efficiency, and $m_{g}$ is the grain mass. The emerging spectrum is then given by

$$
F_{\lambda}(T)=\frac{\kappa_{\lambda} B_{\lambda}(T) M}{D^{2}},
$$

where $D$ is the distance to the dust and $M$ is the total dust mass (Doty \& Leung 1994). Three compositions were tested

\footnotetext{
13 http://herschel.esac.esa.int/science_instruments.shtml
}

using the grain properties from Weingartner \& Draine (2001) and Laor \& Draine (1993) — pure astronomical silicate (Si), pure graphitic solid $(\mathrm{C})$, and an even mixture of both $(\mathrm{C}+\mathrm{Si})$ - with a standard $a^{-3.5}$ distribution of grain sizes (Mathis et al. 1977, hereafter MRN). Note that at mid-IR wavelengths, the absorption coefficient $\kappa$ is relatively insensitive to grain size. A large range of dust temperatures were sampled for each composition, with the dust mass at a given temperature chosen to minimize the $\chi^{2}$ residual between the photometric data and synthetic fluxes found by integrating the model SED over the Spitzer and Herschel filter profiles.

The best-fit models are shown in Figure 3(a), representing $2 \times 10^{-4} M_{\odot}$ of Si dust at $200 \mathrm{~K}, 8 \times 10^{-4} M_{\odot}$ of $\mathrm{C}$ dust at $230 \mathrm{~K}$, and $2 \times 10^{-4} M_{\odot}$ of $\mathrm{C}+\mathrm{Si}$ grains at $245 \mathrm{~K}$. While the $\mathrm{C}$ and $\mathrm{C}+\mathrm{Si}$ models come close to matching the mid-IR SED, the fits differ from the data by $\chi^{2}>30$, suggesting that a single-temperature model is insufficient. We repeated the same exercise using two dust components, where the best-fit mass for each model was determined using the downhill-simplex algorithm amoeba (Press et al. 1992). For a given temperature of the warm component, a large range of cool-dust masses can fit the SED, due to the high upper limits at far-IR wavelengths. Figure 3(b) shows the best-fit models using the lowest masses for each cool-dust component. While the $\mathrm{C}$ and $\mathrm{C}+\mathrm{Si}$ models 

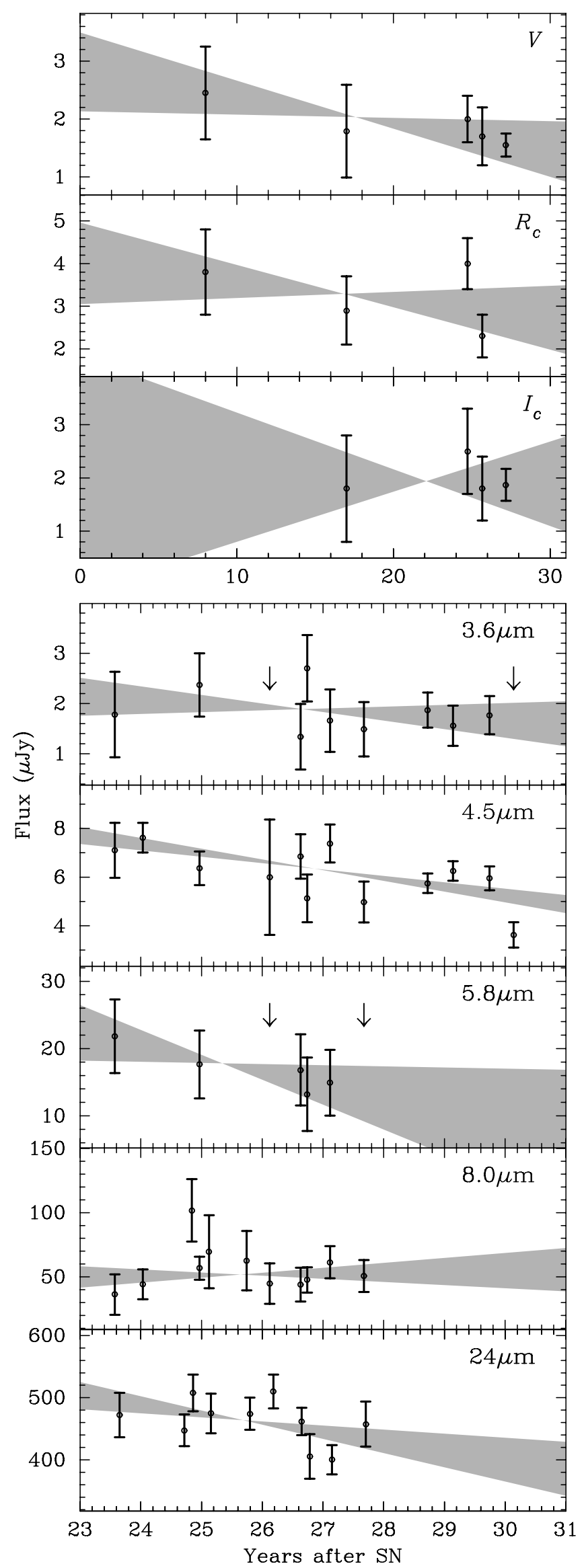

Figure 2. Optical (top) and mid-IR (bottom) light curves of SN 1980K. Wavebands are indicated in the top right of each panel. Gray regions indicate the range of slopes consistent with the data using least-squares fitting.

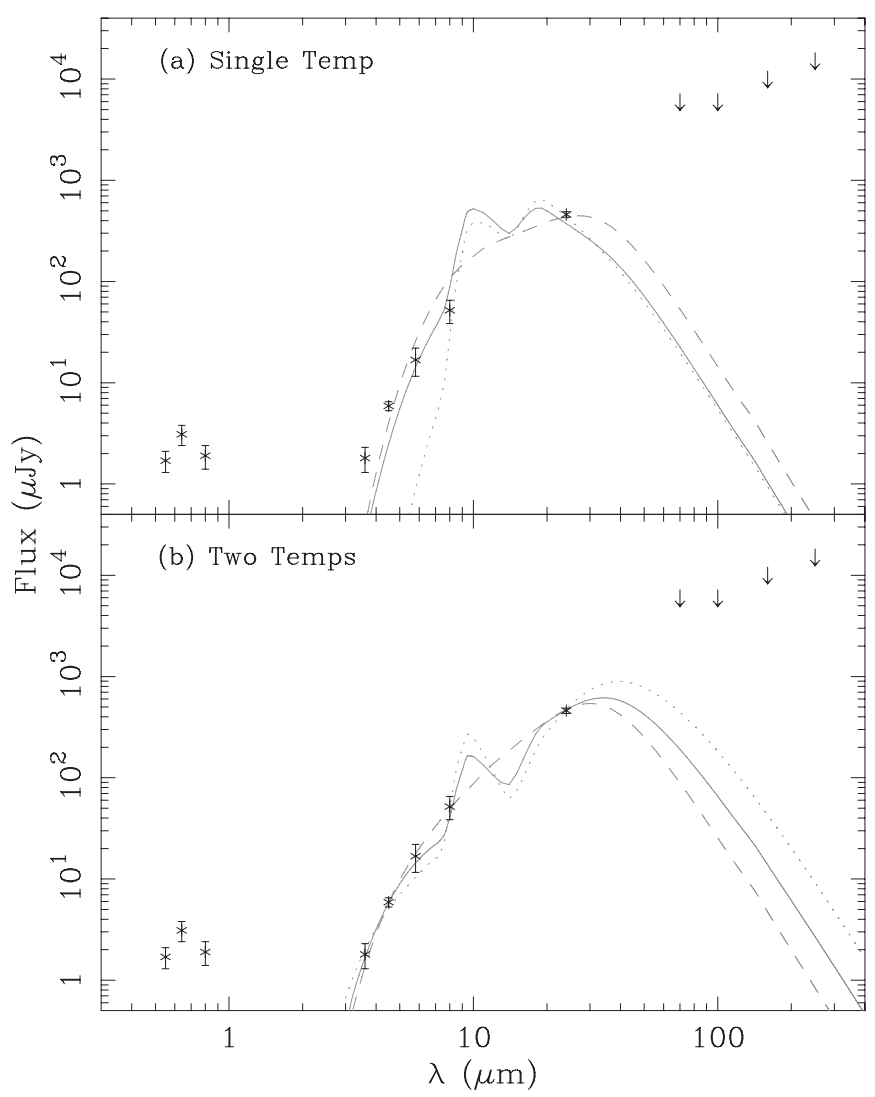

Figure 3. Average SED of SN $1980 \mathrm{~K}$ using data in Table 1, also showing model spectra for simple dust emission models using (a) one and (b) two-temperature components (see Section 3.3). Dotted lines denote silicate dust, dashed lines denote graphitic dust, and solid lines show an equal mixture of both.

Table 2

Results of Two-component Dust Modeling

\begin{tabular}{lcccccccc}
\hline \hline Model & \multicolumn{2}{c}{ Warm Component } & & \multicolumn{2}{c}{ Hottest Cool Dust } & & \multicolumn{2}{c}{ Coldest Cool Dust } \\
\cline { 2 - 3 } & $T_{\text {warm }}(\mathrm{K})$ & $M\left(M_{\odot}\right)$ & & $T_{\text {cool }}(\mathrm{K})$ & $M\left(M_{\odot}\right)$ & & $T_{\text {cool }}(\mathrm{K})$ & $M\left(M_{\odot}\right)$ \\
\hline $\mathrm{C}$ & $300-330$ & $4 \times 10^{-5}$ & & 180 & $10^{-3}$ & & 55 & 3 \\
$\mathrm{Si}$ & $450-480$ & $10^{-4}$ & & 140 & $2 \times 10^{-3}$ & & 45 & 1 \\
$\mathrm{C}+\mathrm{Si}$ & $330-350$ & $10^{-5}$ & & 160 & $10^{-3}$ & & 50 & 3 \\
\hline
\end{tabular}

have $\chi^{2} \sim 1$, the Si models all have $\chi^{2}>21$, suggesting that the dust responsible for the mid-IR emission must have some carbonaceous component.

The dust masses presented in Table 2 represent the instantaneous amount of dust necessary to produce the average SED. Since dust cooling times are short compared to the slow fading of the light curves, we expect that either a small amount of dust is being heated by a source with decreasing energy or the volume of emitting dust is changing with time. In the former case, the dust mass present in the system is roughly that found in this section, while in the latter, the total dust mass is much larger than that which is emitting at any given time. Light echoes are one natural mechanism for illuminating a slowly changing volume of dust with time, where, for our timescales involved, the initial SN light pulse would be heating and scattering off of dust in a large, preexisting circumstellar shell. In the next section, we present a new method for modeling such echoes and explore whether such echoes can reproduce the late-time data. 


\section{LIGHT ECHOES}

\subsection{Formalism}

Light echoes occur when a pulse of light from a variable object (e.g., an SN) interacts with dust and arrives at Earth some time $t$ after the pulse is directly observed. Optical light that scatters from that dust into the line of sight will be observed as a scattered-light echo, while prompt thermal re-emission from dust heated by the pulse will be observed as an infrared (or thermal) echo. Scattered echoes from $\mathrm{SNe}$ have been discussed in, e.g., Chevalier (1986), Sugerman (2003), and Patat (2005), while thermal echoes have been presented in Bode \& Evans (1980), Dwek (1983), and Emmering \& Chevalier (1989). The following is a brief synopsis of the relevant properties of light echoes.

An echo lies on the locus of points equidistant in light travel from the source and observer, i.e., an ellipsoid with the source and observer at its foci. In the neighborhood of the source, this ellipsoid can be approximated by the "echo equation"

$$
z^{2}=\frac{\rho^{2}}{2 c t}-\frac{c t}{2} .
$$

Dust being illuminated at a time $t$ after the $\mathrm{SN}$ is directly observed, located a distance $r$ from the source at an angle $\theta$ from the line of sight, and will have line-of-sight depth $z=r \cos \theta$ and projected distance $\rho=r \sin \theta$ on the plane of the sky. We adopt distances and times in lt-yr and yr, respectively, which simplifies this equation since $c=1$.

Proceeding as in Sugerman (2003), the flux scattered off one dust grain of radius $a$ at position $\boldsymbol{r}$ from the $\mathrm{SN}$ is

$$
d F_{\mathrm{sca}}(\lambda, r, a)=\frac{C_{\mathrm{sca}}(\lambda, a) F(\lambda) \Phi(\mu, \lambda, a)}{4 \pi r^{2}},
$$

where $C=\pi a^{2} Q$ and $Q_{\text {sca }}$ is the grain scattering efficiency, $F(\lambda)$ is the fluence (i.e., time-integrated flux) at the surface of the $\mathrm{SN}$, and $\Phi$ is the scattering phase function for a given scattering angle $\mu=\cos \theta$. We adopt the Henyey \& Greenstein (1941) phase function

$$
\Phi(\mu, \lambda, a)=\frac{1-g^{2}(\lambda, a)}{\left[1+g^{2}(\lambda, a)-2 g(\lambda, a) \mu\right]^{3 / 2}},
$$

with $g(\lambda, a)$ measuring the degree of forward scattering for a given grain. The total flux $F_{\text {sca }}$ integrated over the duration $\Delta t$ of the outburst from a single scattering is found by multiplying Equation (3) by the dust density $n_{d}(r, a)$, integrating over the scattering volume and all grain sizes. If the optical depth of the cloud is low, then one can adopt the single-scattering plus attenuation approximation (SSA; Patat 2005), in which the flux that reaches the dust grain is extinguished by $e^{-\tau_{r}}$ along the radial path from the source to the grain and further by $e^{-\tau_{z}}$ out of the dusty medium along the line of sight to the observer. Implicit in this approximation is that no photons are scattered into the line of sight by other grains of dust within the medium. If the medium consists of a distribution $f(a)$ of different grain sizes such that $n_{d}(r, a)=n(r) f(a)$, the total scattered flux from a volume element $d^{3} r$ arriving at Earth will be

$$
\begin{aligned}
& F_{\mathrm{sca}}(\lambda, \boldsymbol{r}) \\
& \quad=\int \frac{C_{\mathrm{sca}}(\lambda, a) F(\lambda) \Phi(\mu, \lambda, a) n_{0} f(a)}{16 \pi^{2} D^{2} r^{2}}\left(\frac{r_{0}}{r}\right)^{p} e^{-\tau_{r}-\tau_{z}} d a d^{3} r
\end{aligned}
$$

where $n(r)=n_{0}\left(r_{0} / r\right)^{p}$ allows for a power-law dependence of density with radius and $D$ is the distance to the SN. Examples of grain-size distributions include the previously mentioned MRN function and those of Weingartner \& Draine (2001).

A grain of radius $a$ heated to temperature $T$ will radiate

$$
F(\lambda)=C_{\mathrm{abs}}(\lambda, a) B_{\lambda}(T),
$$

where $C_{\mathrm{abs}}$ is the grain absorption (and emission) cross-section and $B_{\lambda}(T)$ is the Planck blackbody function. By analogy with Equation (5), the total thermal emission from all grains within a volume element arriving at Earth will be

$$
\begin{gathered}
F_{\mathrm{th}}(\lambda, \boldsymbol{r})=\int \frac{C_{\mathrm{abs}}(\lambda, a) B_{\lambda}(T) n_{0} f(a)}{4 \pi D^{2}}\left(\frac{r_{0}}{r}\right)^{p} e^{-\tau_{z}} d a d^{3} r . \\
\text { 4.2. Illuminating Spectra }
\end{gathered}
$$

The optical spectrum $F(\lambda)$ that produces scattered-light echoes is taken to be the time-integrated fluence over the first $\sim 100$ days, constructed by interpolating each spectrum onto a regular wavelength grid and integrating them over time using the trapezoidal rule. When considering only scattered light, one may model the echoes using the observed (i.e., reddened) spectrum since it has suffered the same interstellar extinction as any echo flux; however, as we wish to also model the thermal response to the illuminating fluence, the dereddened spectrum must be used. Fabbri et al. (2011) adopt a reddening of $E(B-V)=0.41$ toward the nearby SN 2004et, noting that the foreground Galactic contribution is estimated to be $E(B-V)=0.34$. If SN $1980 \mathrm{~K}$ is located deep within or behind the disk of NGC 6946, one may expect it to have produced largerradius light echoes, as have been observed, for example, around SNe 1987A (Xu et al. 1995), 1993J (Sugerman \& Crotts 2002), and 2003gd (Sugerman 2005). Careful inspection of all optical images shows no such echo features; thus, in our modeling, we consider that while SN $1980 \mathrm{~K}$ may suffer the same extinction as SN 2004et, it may also lie on the near side of its host galaxy and its extinction may be closer to the lower, Galactic value. In what follows, we designate these the "high" and "low" extinction models, respectively. The fluence spectrum, dereddened by the full $E(B-V)=0.41$, is shown in Figure 4(a). Note that since this fluence spectrum ends at $7500 \AA$, it is not possible to model the $I$-band scattered flux of any echoes.

Modeling the thermal echo of an $\mathrm{SN}$ requires considering all the photon heating sources of the dust, which includes not only the long-duration optical pulse but also the preceding short-duration but highly energetic UV pulse, which has been variously modeled by Gezari et al. (2008), Chevalier \& Fransson (2008), Nakar \& Sari (2010), and Rabinak \& Waxman (2011). The blackbody parameters presented in each paper were used to create fluence spectra over the first $\sim 2.3$ days, as shown in Figure 4(b). Although the Chevalier \& Fransson (2008) model was proposed for $\mathrm{SNe} \mathrm{Ib} / \mathrm{c}$, its fluence spectrum describes a reasonable average between the other three models considered; therefore, we adopt this spectrum as describing the initial UV pulse.

\subsection{Constraints}

Figure 1(a) shows that there is no resolved flux around SN $1980 \mathrm{~K}$, and examination of the central source shows that it is consistent with a point source; thus, any echo must come from material such that its flux remains unresolved. Experimentation with marginally resolved artificial sources shows that an echo 

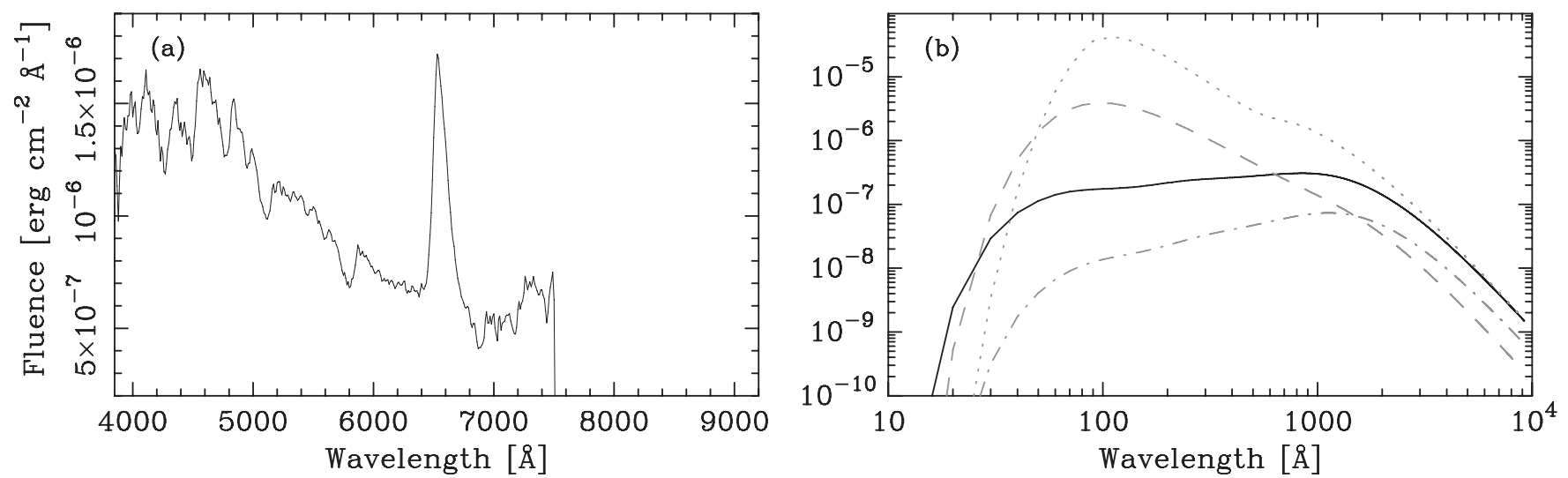

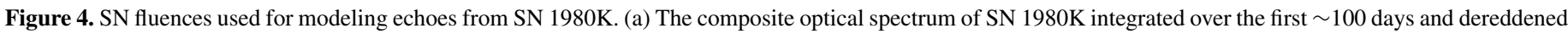

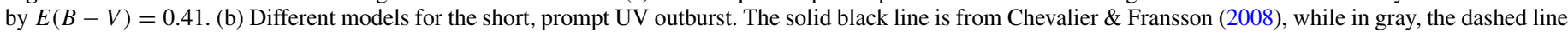
is from Nakar \& Sari (2010), dot-dashed from Rabinak \& Waxman (2011), and dotted from Gezari et al. (2008).

can remain unresolved with a projected distance $\rho$ up to 1.2 times the FWHM of the PSF, which for the WF2 chip yields $\rho \lesssim 0^{\prime} .2$ or roughly 19 lt-yr. At $t=28 \mathrm{yr}$, an echo would be at $z \sim-7$ lt-yr and $r \sim 20$ lt-yr. For an echo to be present $t \mathrm{yr}$ after outburst, material must be at least $t / 2$ lt-yr from the source. Since there is no other spatial information available, we will model the echo as arising from a simple spherical shell with outer radius between 14 and 20 lt-yr. Chevalier \& Emmering (1989) argue that red-supergiant winds expanding into ambient interstellar media will have radius of order $16 \mathrm{lt}-\mathrm{yr}$, consistent with these constraints.

Since the average light curves presented in Figure 2 are monotonically fading in almost all wavebands, we opt to model the system using the average optical and mid-IR data from 23 and 28 yr after outburst, which cover the period of multiwaveband Spitzer observations. Fluxes and their associated uncertainties for each epoch were taken as the least-squares fits to each monochromatic light curve and are listed in the two rightmost columns in Table 1. Lacking any temporal data in the far-IR, we assume that the upper limits from Herschel apply throughout the epochs studied.

\subsection{Modeling Echoes}

The optical and thermal echo SED resulting from an SN will be the sum of the light scattered from the short ( $\sim 2$ day) UV and long ( $\sim 150$ day) optical pulses, as well as the thermal emission resulting from the dust being heated by those two input spectra. The UV pulse contributes negligibly to the optical echo; however, both spectra are important sources of heating for thermal emission.

The equilibrium temperature of dust illuminated by spectrum $F(\lambda)$ (and excluding collisional heating, which is reasonable in low-density, slow-moving, unshocked circumstellar media) is given by (e.g., Rowan-Robinson 1980)

$$
4 \int C_{\mathrm{abs}}(\lambda, a) B_{\lambda}(T) d \lambda=\frac{1}{4 \pi r^{2}} \int F(\lambda) C_{\mathrm{abs}}(\lambda, a) e^{-\tau_{r}} d \lambda .
$$

Numerically, the dust temperature for a given grain size $a$ can be calculated using a root-finding algorithm such as Brent's method (Press et al. 1992), which varies $T$ until both sides of Equation (7) are equal. If dust is not heated beyond $\lesssim 1000 \mathrm{~K}$, the majority of its emission will be in the mid-IR and will contribute insignificantly to re-heating of neighboring dust. A thermal echo spectrum can thus be computed as the emission coming from the adjacent parabolic regions illuminated by the $\mathrm{UV}$ and optical pulses at a given time $t$ after outburst by solving Equation (8) at each point along the echo parabola and summing the contributions using Equation (7). Similarly, the scattered optical component is computed by summing Equation (3) in the same regions.

The analytic expressions presented above are only appropriate in the optically thin regime, since multiple scatterings within an optically thick medium return photons to a given path, as well as remove them. Patat (2005) has shown that for a variety of dust geometries the SSA breaks down for optical depths $\tau \gtrsim 0.1$ and that a proper treatment requires Monte Carlo simulations. A variety of dust radiative-transfer Monte Carlo models exist; however, they are numerically expensive, with the result that exploration of a large parameter space of dust geometries and densities to fit a given data set can require tens of thousands of computing hours. A further complication is that of resolution: most three-dimensional Monte Carlo models track photon paths through a spatial grid of cells, making the run time and memory allocation dependent on $N^{3}$, where $N$ is the number of cells in a given dimension. The UV pulse lasts only $\sim 2$ days; however, a star's CSM can span tens of lt-yr. Without an adaptive meshrefinement scheme, the spatial sampling needed to describe the dust's behavior in this narrow echo requires a prohibitively high number of grid cells.

It is for these reasons that we have developed the QuickSAND semi-analytical model presented in the Appendix, which approximates the results of Monte Carlo dust radiative-transfer models in homogenous media to a high degree of accuracy. For an alternative semi-analytic model, please see Városi \& Dwek (1999, hereafter VD99).

To test whether the proposed scattered and thermal echoes can reproduce the broadband photometry of SN 1980K, we ran the QuickSAND model on a large parameter space of homogenous spherical shells, where each possible shell is described by an inner radius $r_{0}$, thickness $b$, density power-law index $p$ (Section 4.1), and carbonaceous-dust fraction $c$ (such that the amount of silicate dust is $1-c)$. Values of $r_{0}$ and $b$ were tested every 0.5 lt-yr such that $r 0+b<20$ lt-yr (Section 4.3), with $p=0$ and 2 and $c=1 / 2,3 / 4$, and 1 (recall that modeling from Section 3.3 showing silicate-dominated dust does not reproduce the SEDs). As noted in Sugerman (2003), one can use the observed spectrum when modeling a scattered-light echo since the observed spectrum has already suffered the appropriate extinction along the line of sight to the observer. However, the 

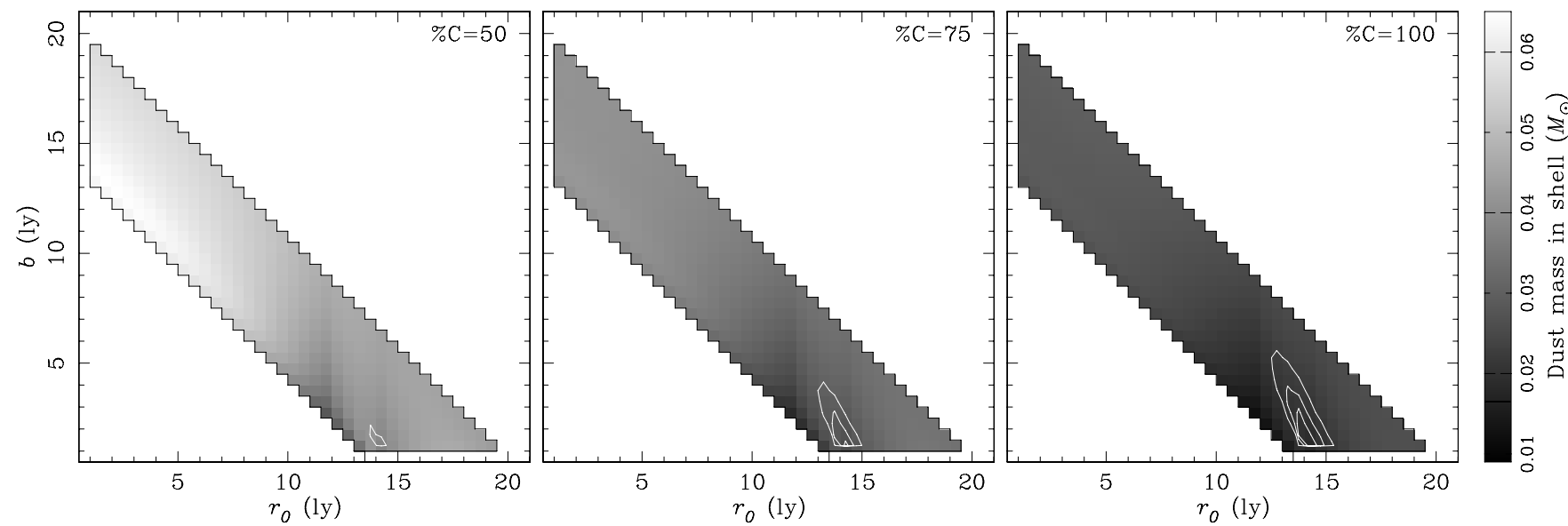

Figure 5. Results of QuickSAND modeling of optical and thermal echoes to reproduce the average photometry of SN $1980 \mathrm{~K}$ at 23 and 28 yr after outburst (Table 1). Each panel shows in gray scale the dust mass that best fits the data for a constant-density $(p=0)$, low-extinction model with the given values of inner-shell radius $r_{0}$, shell thickness $b$, and carbonaceous-dust fraction $c$. The wedge at right shows the scaling between shading and the shell's dust mass. Overplotted on each panel are contours of constant reduced $\chi^{2}$ at intervals of $\chi_{\text {red }}^{2}=0.5$, with the outermost tracing $\chi_{\text {red }}^{2}=2$. The results for high extinction are nearly indistinguishable, with the contour interval changed to unity and the outermost contour tracing $\chi_{\text {red }}^{2}=4$. In the neighborhood of the good fits, the $p=2$ models are also nearly indistinguishable.

extinguished spectrum is inappropriate for heating of dust as the dust receives the unextinguished light. The QuickSAND SEDs were constructed using the unextinguished optical and UV bursts in Figure 4 and then reddened by $E(B-V)=0.42$ to compare against data.

Adopting the same grains as were used in Section 3.3, we used Brent's method to determine the dust density that minimizes the $\chi^{2}$ residuals between the QuickSAND model SEDs and data at both 23 and $28 \mathrm{yr}$ (Table 1) for each set of input parameters. This is another advantage of our semi-analytic model over full radiative-transfer codes, since QuickSAND can automatically find the best-fit density rather than the user having to modify it by hand through trial and error. Another option is to allow such models to find the best fit of all parameters, by using a multidimensional minimization scheme such as the downhill simplex; however, there are many potential problems with this approach, namely, the minimization routine can settle into local minima, the output does not provide uncertanties or ranges of parameter space that fit data, and the runtime is significantly longer than stepping through a grid of parameters while minimizing only one (e.g., density).

The results for the low-extinction model are summarized graphically in Figure 5, which shows in gray scale the dust mass that best fits the data for differing values of $r_{0}, b$, and dust composition, assuming constant density with radius $(p=0)$. Overplotted in white are contours of reduced $\chi^{2}$ (i.e., $\chi^{2}$ per degree of freedom) at intervals of $\chi_{\text {red }}^{2}=0.5$ with the outermost contour tracing $\chi_{\text {red }}^{2}=2$. The best-fit models are thin shells with $r_{0} \sim 14$ lt-yr, $b=1-4$ lt-yr, a medium dominated by carbonaceous dust, and a total dust mass of $0.02-0.05 M_{\odot}$. As normalized by Weingartner \& Draine (2001), the MRN grain distribution corresponds to a gas-to-dust mass ratio of $\sim 150$, which yields a total shell mass of $\sim 3 M_{\odot}$.

Results for the higher-extinction model are nearly indistinguishable, except that the contours are spaced at intervals of $\chi_{\text {red }}^{2}=1$ with the outermost contour tracing $\chi_{\text {red }}^{2}=4$. For both low- and high-extinction cases, the $p=2$ models also yield nearly indistinguishable results in the neighborhood of the good fits. In all models, the $V$-band optical depths of the good fits are $\lesssim 10^{-3}$, i.e., the proposed CSM is optically thin. The SEDs for the best-fit low- and high-extinction models are plotted against the data in Figure 6.

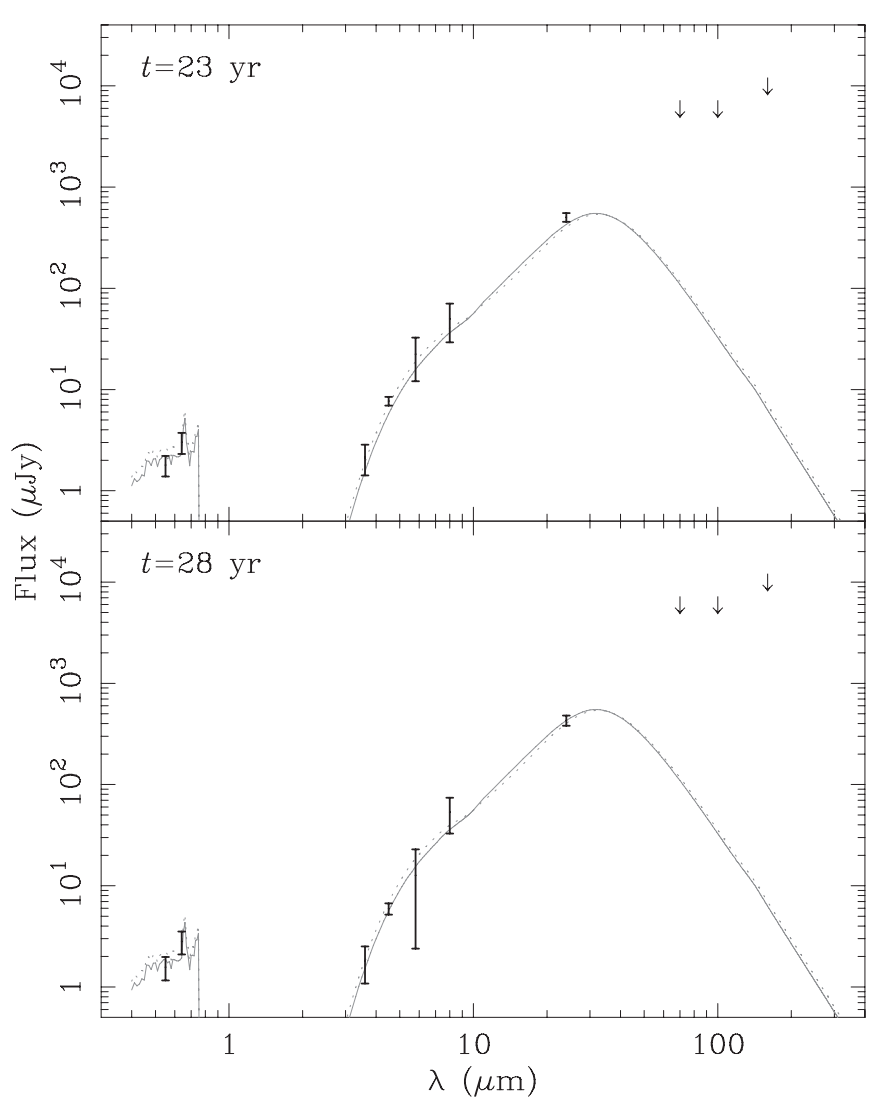

Figure 6. Best-fit high-(solid) and low-extinction (dotted) QuickSAND models of the average optical and mid-IR fluxes at 23 (top) and $28 \mathrm{yr}$ (bottom) after outburst. Both models are for a shell with $r_{0}=14 \mathrm{lt}-\mathrm{yr}, b=1 \mathrm{lt}-\mathrm{yr}$, and $c=1.0$, with $M_{d}=1.5 \times 10^{-2} M_{\odot}$ and $\chi_{\text {red }}^{2}=1.6$ in the high-extinction case and $M_{d}=1.8 \times 10^{-2} M_{\odot}$ and $\chi_{\text {red }}^{2}=1$ for low extinction.

Such a structure may be the contact discontinuity between the star's winds and the ambient interstellar medium (ISM; also sometimes called a wind-blown bubble). The size scale, mass, and dust-grain properties are roughly consistent with the contact discontinuity around SN 1987A reconstructed by Sugerman et al. (2005), which was found to be a pinched prolate spheroid, with polar and equatorial axes of 20 and 11 lt-yr, 


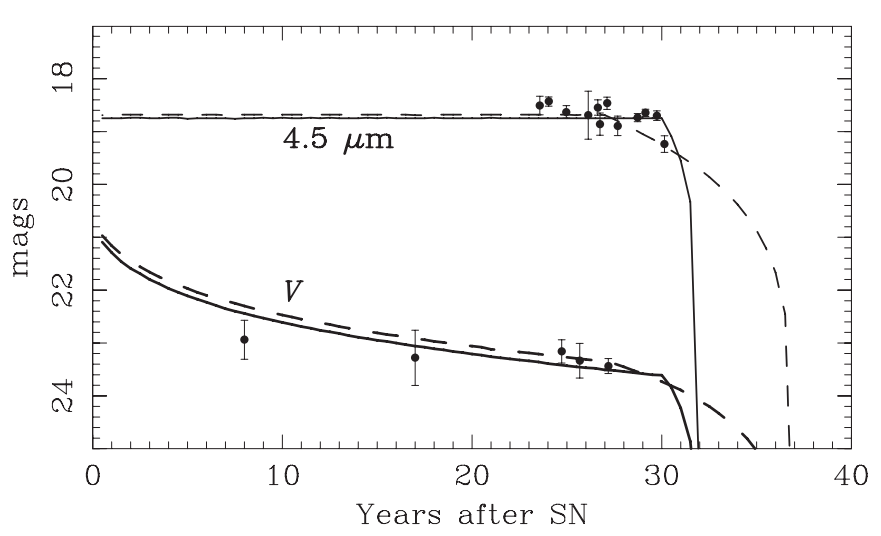

Figure 7. Light curves in $V$ (bottom) and at $4.5 \mu \mathrm{m}$ (top) of echoes off of thin (solid) and thick (dashed) spherical shells that fit the observed data, along with the observed data in both wavebands.

shell thickness $\sim 4$ lt-yr, and total mass $\gtrsim 1.2 M_{\odot}$, assuming a gas-to-dust ratio of $\sim 500$. Recall that SN 1987A is also surrounded by a complex CSM consisting of three illuminated rings and a dusty peanut-shaped structure, located $\sim 1-2$ lt-yr from the progenitor. If SN 1987A is indicative of the massloss structures expected around high-mass stars, then a large wind-blown bubble (representing main-sequence and/or early red-supergiant winds) is expected to be accompanied by inner circumstellar structures that were formed shortly before core collapse. Indeed, Dwek (1983) invoked thermal echoes from such inner material to explain early-time IR excesses from SN 1980K.

The deduced structure could also be consistent with an evolved version of the type of shell found around luminous blue variables such as AG Car, He 3-591, and Sk -69 279, with respective shell dimensions of $6.5 \times 4.5 \mathrm{lt}-\mathrm{yr}, 1.6 \times 1.6 \mathrm{lt}-\mathrm{yr}$, and $20.2 \times 14.7$ 1t-yr. (Weis 2011). The nebulae around the massive central stars of the AG Car and He 3-591 nebulae have been estimated to have cool dust masses of $0.25 M_{\odot}$ and $1 M_{\odot}$, respectively (Voors et al. 2000). The mass-loss rate of AG Car has been estimated to be in the range (1.5-3.7) $\times 10^{-5} M_{\odot} \mathrm{yr}^{-1}$ (Barlow 1991; Groh et al. 2009), comparable to the mass-loss rate estimated for the immediate progenitor of SN 1980k by Dwek (1983). The mid-IR spectrum of AG Car is featureless apart from a weak $11.3 \mu \mathrm{m}$ polycyclic aromatic hydrocarbon emission band (Voors et al. 2000; Rajagopal et al. 2007), also consistent with the dust properties found here to be needed to match the spectrum of the light echo emission around SN 1980k. Regrettably, the observations of and constraints from all echoes are insufficient to warrant a more thorough morphological investigation of these proposed inner and outer circumstellar echoes.

As discussed in Section 4.3, an echo will pass out of a spherical shell of size $t / 2$ lt-yr in $t \mathrm{yr}$; thus, it is important to note that the best-fit models imply that the echo left the CSM shortly after $t=30 \mathrm{yr}$. Figure 7 shows the expected $V$ and $4.5 \mu \mathrm{m}$ light curves for thin $(r 0=15, b=1)$ and thick $(r 0=13.5, b=5)$ shells that are consistent with the data. Note the precipitous drop in flux in the thin shell, as compared to the slow fading in thicker shells. Taken on its own, the drop observed in the $4.5 \mu \mathrm{m}$ datum from $t=30.1 \mathrm{yr}$ (see also Figure 2) is consistent with the light curve expected from the thin-shell model. It should be emphasized that such a drop in the light curve after $30 \mathrm{yr}$ was not fit during our modeling. Obviously, future observations in the optical and mid-IR are required to ensure that the $\mathrm{SN}$ is actually

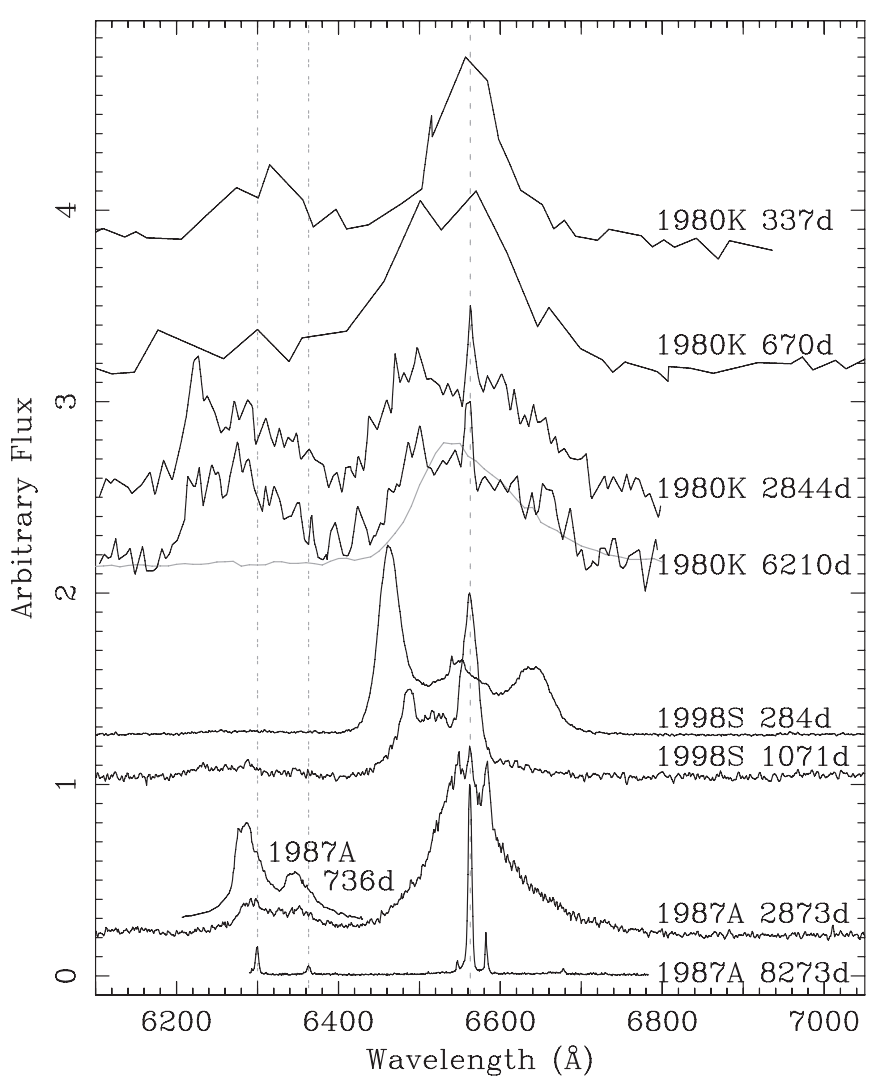

Figure 8. Spectra of SN 1980K (top four), SN 1998S (middle two), and SN 1987A (bottom three) at the dates indicated and scaled to arbitrary flux. In gray under the SN 1980K spectrum at day 6210 is plotted the estimated fluence of the SN from Figure 4(a) as it would appear in an echo when scattered by MRN dust. The dashed thin gray lines show the rest wavelengths for $\mathrm{H} \alpha$ and the [O I] $\lambda \lambda 6300,6363$ doublet.

fading; the epoch of this fading and its duration will serve to disentangle the broad range of acceptable models in Figure 5. Still, with the data in hand it appears that light echoes from a thin shell with inner radius around 14-15 lt-yr are an excellent explanation for the multiband observations of SN 1980K.

\section{COMPARISON WITH PREVIOUS ANALYSES}

As noted in Section 1, Fesen \& Becker (1990), Fesen \& Matonick (1994), and Fesen et al. (1995, 1999) have reported a slowly varying complex of highly blueshifted $\left(v_{r} \lesssim\right.$ $7000 \mathrm{~km} \mathrm{~s}^{-1}$ ) $\mathrm{H} \alpha$ and [O I] $\lambda \lambda 6300,6363$ emission lines. Representative spectra are reproduced at the top of Figure 8. Fesen $\&$ Becker (1990) ruled out these high-velocity features as being due to a light echo, since such features were not seen in early spectra. Instead, they proposed that such features might arise from ejecta shocking preexisting circumsteller material. It is therefore important to consider whether the observational signatures of such an impact are consistent with our proposed light-echo scenario in explaining the past few decades of evolution.

The only very young supernova remnant that has been visually confirmed to have ejecta-CSM impact is SN 1987A, in which the forward blast has been lighting up "hot spots" around the inner equatorial ring or "ER" (e.g., Sugerman et al. 2002; France et al. 2010). Representative spectra from roughly the same epochs as the very late time spectra from SN 1980K are shown at the bottom of Figure 8. The day 2873 spectrum is an HST Cosmic-Origins Spectrograph observation of the entire 
inner ring, first hot spot, and ejecta taken on 1995 January 8, and the day 8723 spectrum is of the ring and a few bright hot spots taken with the Space Telescope Imaging Spectrograph on 2009 October 21. Both spectra were extracted from pipelinecalibrated data taken from the $H S T$ archive. Note the narrow $\mathrm{H} \alpha$, $[\mathrm{N}$ II], and [O I] lines arising from the unshocked ring material. Whether including the entire ring and impact system (day 2873) or only a subset (day 8273), these spectra do not show the very high blueshift emission lines seen in SN 1980K.

In the plane-parallel approximation (Sgro 1975), the transmitted shock velocity $v_{t}$ when impacting media with overdensity $\delta$ is

$$
v_{t}=v_{e} \sqrt{\frac{\beta}{\delta}}
$$

where $v_{e}$ is the ejecta shock speed and $\beta=6\left(1+1.701 \delta^{-1 / 2}-\right.$ $\left.0.4018 \delta^{-1}+0.2274 \delta^{-3 / 2}-0.0874 \delta^{-2}\right)^{-2}$ (Borkowski et al. 1997) measures the pressure differential pre- and post-shock. While the SN 1987A ejecta are moving at $\sim 3500 \mathrm{~km} \mathrm{~s}^{-1}$, the shocks transmitted into the ER travel at significantly lower speeds $\left(\sim 200 \mathrm{~km} \mathrm{~s}^{-1}\right)$, producing lines that are far narrower and less Doppler-shifted (Michael et al. 2000; Pun et al. 2002) than those in SN 1980K. It is therefore unclear whether the high-velocity features in SN 1980K can be adequately explained by an impact with slow-moving CSM.

Nonetheless, if this impact is indeed underway, then dust present within the CSM will be heated either collisionally or radiatively within the shocked and/or post-shocked gas. Bouchet et al. (2006) have investigated these mechanisms for thermal emission from the hot spots in SN 1987A and find that the dust is most likely heated by radiation from post-shocked gas, has a density $\sim 10^{-8} \mathrm{~cm}^{-3}$, and is heated to $\sim 150 \mathrm{~K}$. The SN 1980K CSM models from Dwek (1983) yield dust and gas densities similar to those in the ER of SN 1987A, while spectral analyses from, e.g., Fesen et al. (1999) suggest that the ejecta could be expanding up to $\sim 5000 \mathrm{~km} \mathrm{~s}^{-1}$. Since post-shock gas temperature scales as $v_{t}^{2}$ (see Equation (10) below), dust that is radiatively heated by that gas will have temperature $T_{d} \propto \sqrt{v_{t}}$ (Borkowski et al. 1997; Bouchet et al. 2006). We can therefore approximate the ejecta-CSM impact as heating dust to $\lesssim 300 \mathrm{~K}$, which is close to the range of warm dust found in our twotemperature fits in Section 3.3. A single "skin" of $300 \mathrm{~K} \mathrm{MRN}$ dust that is $10^{16} \mathrm{~cm}$ thick at the $\sim 3 \times 10^{17} \mathrm{~cm}$ radius of the Dwek (1983) model emits up to an order of magnitude more flux at $8 \mu \mathrm{m}$ than has been observed. If one adopts the twoshock model from Pun et al. (2002), in which slow and fast shocks are transmitted into the CSM based on the geometry and density of the impacted medium, and if one makes the reasonable assumption that the CSM is not a complete spheroid (i.e., thermal emission is not coming from all $4 \pi \mathrm{sr}$ ), then an ejecta impact could explain the average mid-IR SED, provided that the slow shock component has a velocity $\lesssim 25 \%$ that of the fast shocks.

A complete investigation of the radiation expected from ejecta-CSM impact requires time-dependent gas and dust radiative-transfer shock modeling, which is outside the scope of this paper. However, we can compare the heating and cooling timescales to estimate the overall energetic evolution of such a system. Again taking SN 1987A as our example, Pun et al. (2002) found that a forward blast traveling at $\sim 3500 \mathrm{~km} \mathrm{~s}^{-1}$ from an $\mathrm{H}$ II region with $n \sim 150 \mathrm{~cm}^{-3}$ into CSM with $n \gtrsim 10^{4} \mathrm{~cm}^{-3}$ will produce shocks traveling at $v_{t} \sim 250 \mathrm{~km} \mathrm{~s}^{-1}$ and heating post-shock gas to an equilibrium temperature of $\sim 10^{6} \mathrm{~K}$. Using their cooling-time approximation

$$
t_{\mathrm{cool}}=2.2\left(\frac{2 \times 10^{4} \mathrm{amu} \mathrm{cm}^{-3}}{\mu_{A} n_{0}}\right)\left(\frac{v_{t}}{250 \mathrm{~km} \mathrm{~s}^{-1}}\right)^{3.8} \mathrm{yr},
$$

where $n_{0}$ is the pre-shock density of the impacted material and $\mu_{A}$ is the mean atomic weight, we expect radiative cooling times of order 1-2 yr. With similar densities and transmitted shock velocities (preceding paragraph), we expect the cooling timescales of shocked CSM in SN $1980 \mathrm{~K}$ to be of this same order. To explain the high-velocity lines and thermal emission (i.e., seen 10-30 yr after outburst), the ejecta-CSM shocks would have characteristic ages $t_{\text {shock }} \gg t_{\text {cool }}$, meaning that the observed flux levels scale with the surface area of material being shocked. For a complete or partial shell, this would increase with time, which is inconsistent with the observed fading across all wavebands. It is certainly to be expected that the ejecta from SN 1980K are impacting inner CSM; however, it appears that this mechanism can account for (at most) a small fraction of the observed mid-IR flux and may be predominantly responsible for the presence of the narrow systemic emission lines.

The shape and evolution of $\mathrm{H} \alpha$ emission lines from $\mathrm{SN}$ $1998 \mathrm{~S}$ are also indicative of ejecta-CSM interaction (Pozzo et al. 2004); two representative spectra are shown in the middle of Figure 8. Note the early appearance of a triple-peaked $\mathrm{H} \alpha$ profile, believed to arise from impact of ejecta with an edge-on ring (Gerardy et al. 2000). By day 658, the red wing of this profile had disappeared, likely due to preferential extinction of emission from receding ejecta by dust that had condensed within it. This effect was also seen in the [O I] lines in SN 1987A by Lucy et al. (1989), the spectrum of which is shown for day 736 in Figure 8. At later times, the emergence of a strong, central peak, slowing of the blueshifted line, and lack of any red emission can be interpreted by the "clumpy wind" model of Chugai \& Danziger (1994), in which intermediate-width spectral features seen at late times arise from shocked clumps of dense wind.

Although SN 1998S is a different Type (IIn) of SN, The $\mathrm{H} \alpha$ profile in its late spectrum bears enough resemblance to those of the $\mathrm{H} \alpha$ and [OI] lines in the very late spectra of SN $1980 \mathrm{~K}$ to suggest that the same mechanism may be at play. One concern with invoking ejecta shocking a clumpy progenitor wind is that slow-moving clumps must be accelerated to high velocities to explain the observations, and simulations of ejectaclump impacts to model the hot spots in SN 1987A result in post-shock gas moving at hundreds of $\mathrm{km} \mathrm{s}^{-1}$ rather than many thousands, as needed to match the systemic speeds of the high-velocity emission lines. We question whether these high-velocity features may instead arise from reverse shocks traveling through high-velocity and high-density clumps of metal-rich ejecta. These have long been predicted to form in twodimensional simulations (Chevalier \& Klein 1978) and recently observed in fully three-dimensional simulations of asymmetric core-collapse explosions (Hammer et al. 2010). Furthermore, such clumps are common in evolved remnants such as Cas A (Fesen et al. 2011), N63A (Warren et al. 2003), and G292.0+1.8 (Ghavamian et al. 2005).

As above, the time-dependent modeling of such a system is outside the scope of this paper; however, it is instructive to considering whether emission from such clumps is expected to evolve over the observed multi-year timescales (Fesen et al. 1999). We adopt the homologous-expansion model of Chevalier (1982), in which the size $R$ of a homologously expanding medium scales as $R \propto t^{(n-3) /(n-s)}$, where $n$ and $s$ are the 
density power-law indices of the expanding and stationary media, respectively. Making the typical assumption that $n=9$ and $s=2$ for ejecta expanding into a low-density CSM, the size of an ejecta clump will grow roughly as $t^{6 / 7}$. In the Hammer et al. (2010) simulation, oxygen-rich clumps (which would produce the observed high-velocity [O I] lines) have sizes around $\sim 2 \times 10^{11} \mathrm{~cm}$ and gas densities $\sim 10^{16} \mathrm{~cm}^{-3}$ about $9000 \mathrm{~s}$ after core collapse. At an age of $\sim 300 \mathrm{yr}$ (roughly that of Cas A), these clumps would grow to $R \sim 3 \times 10^{16} \mathrm{~cm}$ and $n \sim 100 \mathrm{~cm}^{-3}$, which are in excellent agreement with size scales of (1-2) $\times 10^{16} \mathrm{~cm}$ and densities of $100-300 \mathrm{~cm}^{-3}$ for metal-rich knots in Cas A (Fesen et al. 2001; Docenko \& Sunyaev 2010).

At ages of 15 and $30 \mathrm{yr}$, such clumps would have sizes $R \sim 1.5 \times 10^{15}$ and $\sim 4 \times 10^{15} \mathrm{~cm}$ and densities $n \sim 10^{4}$ and $\sim 2 \times 10^{3} \mathrm{~cm}^{-3}$, respectively, which correspond roughly to the epochs of spectroscopic observation of high-velocity lines and mid-IR photometry, respectively. An oxygen-dominated clump $\left(\mu_{A} \sim 18\right)$ traveling outward at thousands of $\mathrm{km} \mathrm{s}^{-1}$ will have radiative cooling times $\ll 1$ yr using Equation (10), which is much shorter than the crossing times of 1-4 yr for the expected range of transmitted shock velocities. As above, a shocked clump will emit over its crossing time, which corresponds well with the timescales during which the [OI] lines have been observed to change.

An oxygen-rich clump with the properties above will have a total mass $\sim 10^{-5} M_{\odot}$. If silicate dust within this clump forms with an efficiency of $1 \%$, its thermal energy output at a few hundred $\mathrm{K}$ will be over an order of magnitude fainter than the observed mid-IR fluxes. However, this assumes that the entire clump is radiating, and given the short cooling times, it is likely that the mid-IR emission from a given clump at a given time will be significantly less. We cannot rule out the possibility that a number of such clumps with a variety of different preshock densities and geometries could provide the temperature range needed to fit the thermal SEDs. As with the ejecta/CSM impact, however, one expects that the number of emitting loci will increase with time as more clumps are shocked, in contrast to our observations of a slow mid-IR decline. We posit that while high-velocity clumps may explain the observed emission-line structures, they contribute only a small fraction of the mid-IR emission.

Behind the day 6210 spectrum of SN 1980K (Figure 8), we have plotted the fluence of SN 1980K (Section 4.2) as scattered by the best-fit thin dust shell. Note that this echo spectrum can account for most of the very broad $\mathrm{H} \alpha$ profile, as well as the faint continua present in all the late-time spectra. Thus, we believe that the proposed scattered and thermal echoes, in combination with high-velocity, oxygen-rich ejecta clumps, are a reasonable explanation for the observed photometric and spectroscopic very late time behavior of SN 1980K.

\section{CONCLUSIONS}

Below we summarize the main results of this paper.

1. We have observed SN $1980 \mathrm{~K}$ in optical and mid-IR wavebands from 23 to $30 \mathrm{yr}$ after outburst. The SN was not detected in the far-IR bands of Herschel.

2. The optical light curves are consistent with fading that has been previously reported, and the mid-IR light curves all show the same trend.

3. The instantaneous dust mass needed to explain the midIR SEDs is $\gtrsim 10^{-3} M_{\odot}$, with the presence of as much as a few $M_{\odot}$ of cold dust possible given the large upper limits in the far-IR.
4. We have presented a new method for computing the effective optical depth of a scattering and absorbing medium (Equation (A12)) and an empirical correction that is independent of the particular grain species or properties (Equation (A14)).

5. When used to characterize the line-of-sight extinction to a particular location within a homogenous dusty medium, these expressions for the effective optical depth in the equations of scattering (Equation (5)) and radiative balance (Equation (7)) yield dust temperatures and emerging scattered and thermal spectra that are correct to within $\sim 10 \%$ for optically thin and to within a factor of two for optically thick media when compared to solutions using the Monte Carlo radiative-transfer code MOCASSIN. In practice, this "QuickSAND" runs up to a few hundred times faster than a traditional Monte Carlo dust radiative-transfer code.

6. We use the QuickSAND model to compute the thermal and scattered echo from an SN over a large parameter space of dust composition, density power laws, and inner- and outer-shell radii and find that the observed optical and midIR SEDs are consistent with echoes from a thin CSM shell of inner radius $\sim 14$ lt-yr and mass $\lesssim 0.02 M_{\odot}$ of carbonrich dust. This is consistent with the size expected for the contact discontinuity between a massive star's winds and the ambient ISM and with the size, composition, and mass of the contact discontinuity found around SN 1987A.

7. Previous observations of SN $1980 \mathrm{~K}$ have revealed highly blueshifted [O I] and $\mathrm{H} \alpha$ lines, interpreted as arising in impact of the SN blast with preexisting CSM. "Back of the envelope" considerations suggest that these lines may arise instead in metal-rich clumps of ejecta, while the ejecta-CSM impact may give rise instead to narrower lines observed at their rest wavelengths, and that neither mechanism is likely to produce sufficient thermal emission to explain the observed mid-IR SEDs.

The quality of the light-echo modeling presented here is not exclusive to MRN dust for a spherical shell. For example, limiting the largest grains to sizes $\lesssim 0.1 \mu \mathrm{m}$ (as suggested by light-echo studies of inner CSM by Sugerman et al. 2005) yields acceptable fits with nearly the same geometry but a total dust mass of up to $0.06 M_{\odot}$. Certainly one could study an enormous range of grain-size distributions, optical constants, and shell geometries (i.e., ellipsoids, disks, hourglasses, etc.), and we expect that many of these would yield roughly similar fits. Given that this system is unresolved and sparsely sampled in the optical, we must accept the ambiguous result that the total dust mass in the proposed spheroidal wind-blown bubble is $\lesssim 0.1 M_{\odot}$, until another decade of contemporaneous optical and mid-IR observations is available.

In reconstructing the CSM of SN 1987A, Sugerman et al. (2005) found a total of $\lesssim 0.02 M_{\odot}$ of dust (based on the ambiguity of the gas-to-dust mass ratio), with that budget dominated by the large contact discontinuity. While close to the quasi upper limit of $\lesssim 0.1 M_{\odot}$ of dust in the same shell around SN $1980 \mathrm{~K}$, both values are under the total mass of $0.3-3 M_{\odot}$ of dust in the SN 1987A remnant inferred by recent Herschel observations (Matsuura et al. 2011). As shown in Table 2, a large amount of cool dust can reside in the SN 1980K system and remain undetected by Herschel at the depth of the 2010 observations. It remains unclear whether the total dust mass present in this remnant is consistent with that in SN 1987A. Significantly deeper Herschel observations are needed of SN $1980 \mathrm{~K}$ to place more stringent limits on this value. 
The early SN 1980K spectra used in this work are part of the Asiago-Padova Supernova Archive. We thank Annop Wongwathanarat and the authors of Hammer et al. (2010) for generously providing the data from their simulation. We gratefully acknowledge our referee, Fernando Patat, for his critical reading and insightful feedback on the manuscript, as well as bringing the ASA spectra of SN 1980 K to our attention. Support for B.E.K.S. for this work was provided by Hubble Space Telescope award GO-10607. This work is based in part on data obtained with the Spitzer Space Telescope, which is operated by the Jet Propulsion Laboratory, California Institute of Technology, under a contract with NASA; with the NASA/European Space Agency Hubble Space Telescope, which is operated by the Association of Universities for Research in Astronomy, Inc. (AURA), under NASA contract NAS 5-26555; with the Gemini Observatory, which is operated by AURA under a cooperative agreement with the NSF on behalf of the Gemini partnership; and with Herschel, an ESA space observatory with science instruments provided by European-led Principal Investigator consortia and with important participation from NASA. IRAF is distributed by the National Optical Astronomy Observatories, which are operated by the Association of Universities for Research in Astronomy, Inc., under cooperative agreement with the National Science Foundation.

\section{APPENDIX}

\section{SEMI-ANALYTIC DUST RADIATIVE TRANSFER}

\section{A.1. Isotropic Scattering}

Following the arguments in Rybicky \& Lightman (1985), consider a homogenous, dusty medium, characterized by absorption and scattering cross-sections $C_{\mathrm{abs}}$ and $C_{\text {sca }}$, respectively. Traditionally, the optical depth $\tau$ to some distance $L$ is defined as $\tau=L / l$, where

$$
l=\frac{1}{C_{\mathrm{abs}}+C_{\mathrm{sca}}}
$$

is the mean-free path inside that medium. Since $\left(C_{\mathrm{abs}}+C_{\mathrm{sca}}\right)$ is the total extinction cross-section $C_{\text {ext }}$, it follows that the fractional absorption

$$
\epsilon=\frac{C_{\mathrm{abs}}}{C_{\mathrm{abs}}+C_{\mathrm{sca}}}
$$

which also gives the probability that a photon path will end with absorption. If the physical size $L$ of the medium is large compared to the mean-free path, then the average number $N$ of mean-free paths that the photon will travel before being absorbed is

$$
N=1 / \epsilon
$$

since $\epsilon$ is the probability of a given step ending in absorption.

The path of a photon in that medium can be expressed as the vector sum $\vec{R}=\sum_{i=1}^{N} \vec{r}_{i}$ of the $N$ scatters taken before absorption (or escape). For isotropic scattering, a photon's path is a random walk, with the well-known results that the average final position $\langle\vec{R}\rangle$ of many photons will be zero, while the average displacement of the photons from their starting point is the rms distance $l_{*}=\sqrt{\left\langle\vec{R}^{2}\right\rangle}$, which expands out to

$$
l_{*}^{2}=\sum_{i=1}^{N}\left\langle r_{i}^{2}\right\rangle+2 \sum_{i=1}^{N} \sum_{j=i+1}^{N}\left\langle\vec{r}_{i} \cdot \vec{r}_{j}\right\rangle .
$$

Since the scattering is assumed to be isotropic, the first term $\left\langle r_{i}^{2}\right\rangle \approx l^{2}$ while the cosines in the cross terms average to zero, yielding the expected result $l_{*}=l \sqrt{N}$. Combining this with Equations (A1)-(A3) yields $l_{*}^{2}=l^{2} / \epsilon$ or

$$
l_{*}=\left[C_{\mathrm{abs}}\left(C_{\mathrm{abs}}+C_{\mathrm{sca}}\right)\right]^{-1 / 2} .
$$

The optical thickness of a medium is approximately the number of mean-free paths required to escape, i.e., $\tau=L / l$ as defined above. For an optically thick medium where the probability of absorption is non-trivial, one must redefine optical thickness as the ratio of the physical size $L$ of the medium to the average distance $l_{*}$ between creation and destruction of a photon. As such, $l_{*}$ is often called the "effective mean path" and the resulting effective optical depth

$$
\begin{aligned}
\tau_{*} & =\frac{L}{l_{*}} \\
& =\sqrt{\tau \tau_{\mathrm{sca}}} \\
& =\frac{\tau}{\sqrt{N}},
\end{aligned}
$$

where the optical depths due to absorption, scattering, and extinction are $\tau_{\mathrm{abs}}=L C_{\mathrm{abs}}, \tau_{\mathrm{sc}}=L C_{\mathrm{sca}}$, and $\tau=\tau_{\mathrm{abs}}+\tau_{\mathrm{sc}}$, respectively. Defining the scattering albedo $\omega=\tau_{\mathrm{sc}} / \tau$, a little algebra yields

$$
\tau_{*}=\tau \sqrt{1-\omega},
$$

which is equivalent to Equation (14) of VD99.

\section{A.2. Non-isotropic Scattering}

If one relaxes the assumption of isotropic scattering, a more generalized expression can be derived by noting that the scattering anisotropy parameter $g=\langle\cos \theta\rangle$, where $\theta$ is the scattering angle between incoming and outgoing photon trajectories. For isotropic scattering, $g=0$, while $g \rightarrow 1$ for pure forward scattering. If $g \neq 0$, the cross terms in Equation (A4), $\left\langle\vec{r}_{i} \cdot \vec{r}_{i+1}\right\rangle \approx g l^{2}$, and by recursion, $\left\langle\vec{r}_{i} \cdot \vec{r}_{j}\right\rangle \approx$ $g^{j-i} l^{2}$. Since $g<1$, the cross terms form a convergent geometric sequence such that

$$
2 \sum_{i=1}^{N} \sum_{j=i+1}^{N}\left\langle\vec{r}_{i} \cdot \vec{r}_{j}\right\rangle=2 g l^{2} \frac{\left(g^{N}-g N+N-1\right)}{(g-1)^{2}} .
$$

Inserting this back into Equation (A4) yields

$$
\frac{l_{*}}{l}=\sqrt{N_{*}(g)}
$$

where

$$
N_{*}(g)=\frac{N-g\left(2+g N-2 g^{N}\right)}{(g-1)^{2}}
$$

and $N$ is defined as in Equation (A3). Note that the limit $N_{*}(g \rightarrow 1)=N^{2}$. Finally, substituting Equation (A10) into Equation (A6) yields the more general expression for effective optical depth

$$
\tau_{*}(g)=\frac{\tau}{\sqrt{N_{*}(g)}} .
$$

Note that Equation (A12) reduces to Equation (A7) for isotropic $(g=0)$ scattering, while for fully forward-scattering particles ( $g=1), \tau_{*}(g) \approx \tau / N$, which is equivalent to Equation (15) of VD99. 

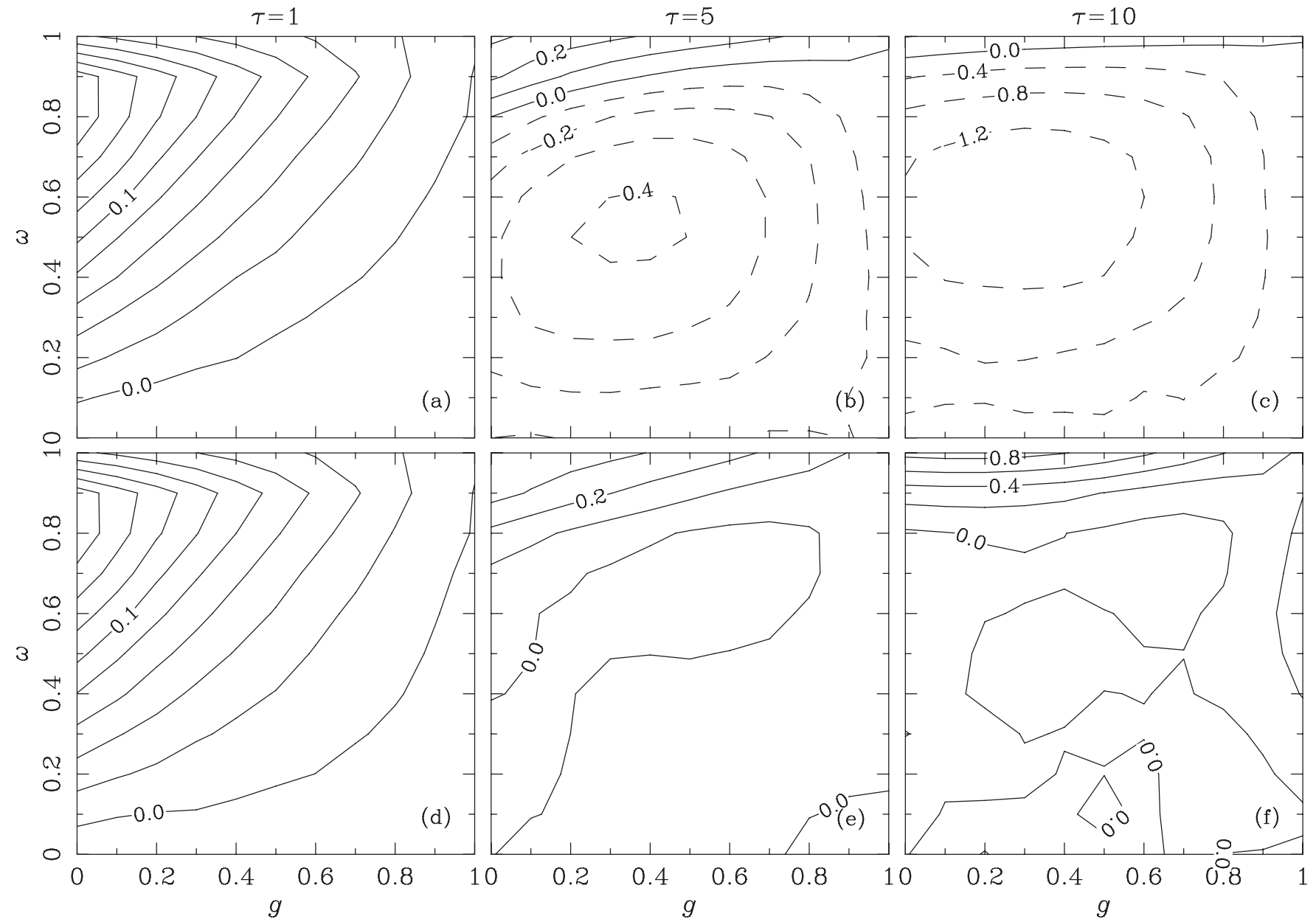

Figure 9. Selected results from testing of the effective optical depth, showing $\tau_{*}-\tau_{\text {eff }}$ for different values of the parameter triplet $\omega, g$, and $\tau$. The top row computes $\tau_{*}$ with Equation (A12). The bottom row includes the empirical correction term $\delta \tau_{*}$ (Equation (A14)). Positive (negative) contours are plotted with solid (dashed) lines at the levels indicated.

\section{A.3. Testing}

We consider the dust radiative-transfer code MOCASSIN (Ercolano et al. 2005) to be the benchmark against which the effective optical depth $\tau_{*}$ can be tested. MOCASSIN selfconsistently computes the radiative transfer of a given energy spectrum $F_{0}(\lambda)$ through a dusty medium composed of userspecified grain properties within a Cartesian grid of cells by following the paths of energy packets (i.e., photons) until the emergent flux has converged to a specified tolerance. Since photons follow random paths, the emergent spectrum $F(\lambda)$ has an inherent variance that depends on the number of photons and the convergence tolerance.

By comparing the emergent and input (or unattenuated) fluxes, the effective optical depth of a given model is defined as

$$
\tau_{\text {eff }}=\ln \frac{F_{0}(\lambda)}{F(\lambda)} .
$$

A grid of models was constructed with $\omega$ and $g$ ranging from 0 to 1 and optical depth $\tau$ from 0.1 to 15 , allowing us to compare $\tau_{*}$ to $\tau_{\text {eff }}$ for each triplet of parameters. Rather than using actual dustscattering properties, each model used uniform values of $C_{\mathrm{abs}}$, $C_{\text {sca }}$, and $g$ for a variety of wavelengths within a spherical shell of inner and outer radii $10^{14}$ and $10^{15} \mathrm{~cm}$ to match the parameter triplet $(\omega, \tau, g)$ being tested. Using a uniform input spectrum, $\tau_{\text {eff }}$ is computed as the average across all wavelengths, providing a measurement that is more robust to the inherent variance of the Monte Carlo process. Selected results for $\tau=1,5$, and 10 are shown in Figure 9. The quality of the approximation $\tau_{*}$ is roughly consistent with those presented in VD99. The most substantial difference is that the residuals $\tau_{*}-\tau_{\text {eff }}$ are well described by an elliptical Gaussian, which is approximately modeled by the empirical expression

$$
\delta \tau_{*}=\left\{\begin{array}{cl}
(0.219 \tau-0.687) e^{-4 \ln 2(\rho / 0.75)^{2}} & \text { if } \tau \leqslant 5.25 \\
7.5 \times 10^{-3} \tau^{2.5} e^{-4 \ln 2(\rho / 0.75)^{2}} & \text { otherwise }
\end{array},\right.
$$

where $\rho=\left((9 / 16)\left(g-g_{0}\right)^{2}+\left(\omega-\omega_{0}\right)^{2}\right)^{-1 / 2}, g_{0}=0.3\left(1+e^{-\tau / 3}\right)$, and $\omega_{0}=0.585\left(1+e^{-\tau / 3}\right)$. The bottom row of Figure 9 shows the result of adding this correction term to $\tau_{*}$, yielding a very accurate approximation of $\tau_{\text {eff }}$ over a very broad range of dust properties in the optically thick regime.

\section{A.4. The QuickSAND Model}

Replacing the optical depth $\tau$ in Equations (3), (7), and (8) with $\tau_{*}+\delta \tau_{*}$ from Equations (A12) and (A14), we can compute the dust temperature and emergent spectra from every point within a homogenous (i.e., non-clumpy) medium. We call this the "QuickSAND" (Quick Semi-ANalytic Dust) radiativetransfer model.

We benchmark tested the QuickSAND model against MOCASSIN by illuminating a spherical shell of inner and outer 


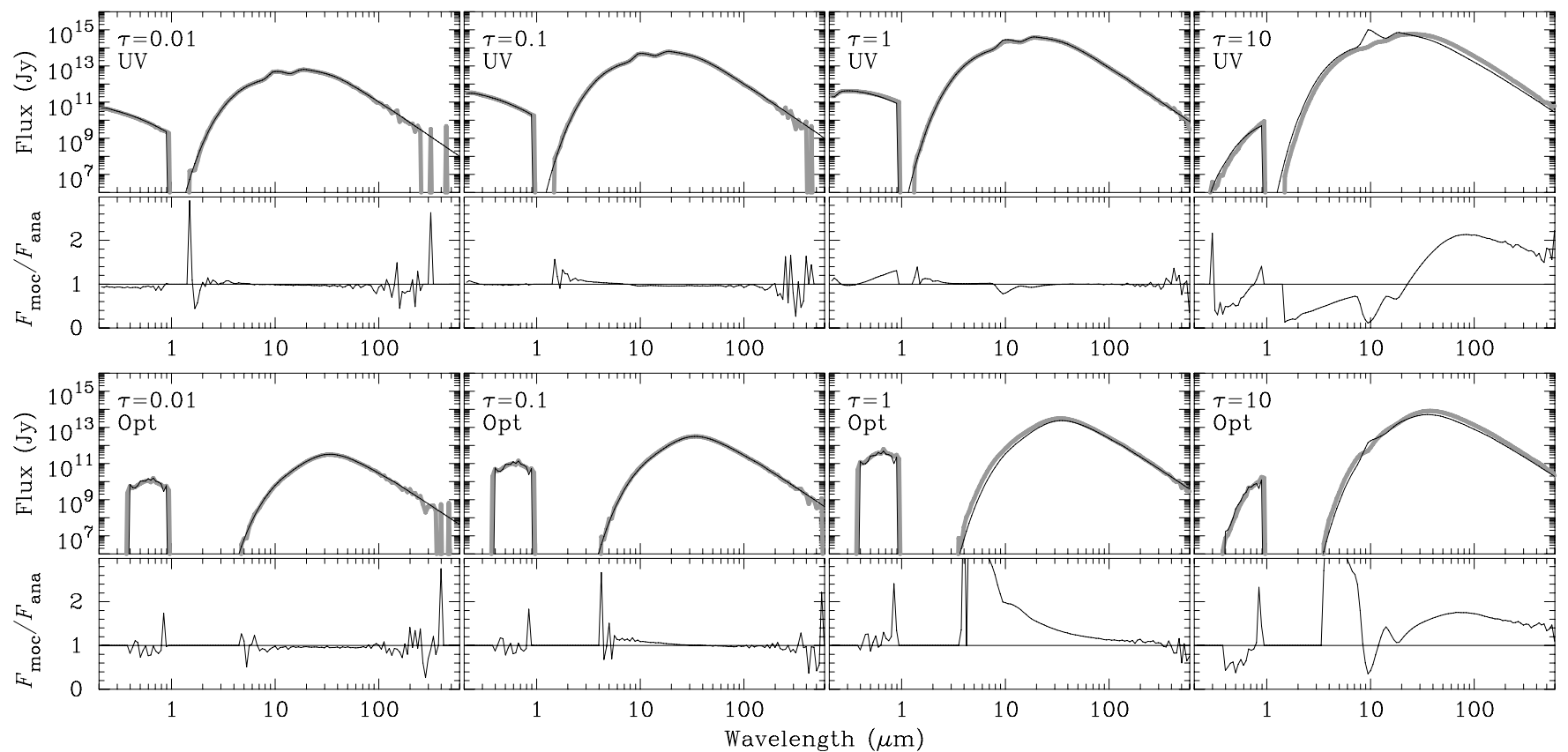

Figure 10. Comparison of our semi-analytic dust radiative-transfer model (QuickSAND) to MOCASSIN for the SN 1980K UV (top row) and optical (bottom row) fluences illuminating spherical shells of optical depth listed at the top left corner of each panel. The SED from MOCASSIN is shown in thick gray and QuickSAND in thin black in the top half of each panel pair. The bottom half of each pair shows the ratio of the two models.

radii of 10 and 15 lt-yr, respectively, composed of an even mixture of carbonaceous and silicate MRN dust (Section 3.3) with constant density and illuminated by the UV and optical fluences in Figure 4. The MOCASSIN models use a reasonably dense cubic grid of 61 cells in each dimension and 128 million energy packets, both of which are needed to ensure that sufficient photons are scattered into the line of sight to provide adequate signal-to-noise ratio in optical scattering and the far-IR. The QuickSAND models sample the shell at roughly double the spatial resolution as MOCASSIN.

Resulting benchmark SEDs from both models are shown in Figure 10 for $\tau_{V}=0.01,0.1,1$, and 10 . The QuickSAND model provides an excellent approximation to the MOCASSIN model at low and intermediate optical depths and is correct to within a factor of two in optically thick media. For comparison purposes, the MOCASSIN model running in parallel on eight processors requires roughly 30 times longer to converge than the QuickSAND model running on a single processor.

\section{REFERENCES}

Barbieri, C., Jr., Bonoli, C., \& Cristiani, S. 1982, A\&A, 114, 216

Barbon, R., Ciatti, F., \& Rosino, L. 1982, A\&A, 116, 35

Barlow, M. J. 1991, in IAU Symp. 143, Wolf-Rayet Stars and Interrelations with Other Massive Stars in Galaxies, ed. K. A. van der Hucht \& B. Hidayat (Dordrecht: Kluwer), 281

Benetti, S. 1989, PhD thesis, Universitá di Padova

Bode, M. F., \& Evans, A. 1980, MNRAS, 193, 21P

Borkowski, K. J., Blondin, J. M., \& McCray, R. 1997, ApJ, 477, 281

Bouchet, P., Dwek, E., Danziger, J., et al. 2006, ApJ, 650, 212

Chevalier, R. A. 1982, ApJ, 258, 790

Chevalier, R. A. 1986, ApJ, 308, 225

Chevalier, R. A., \& Emmering, R. T. 1989, ApJ, 342, 75

Chevalier, R. A., \& Fransson, C. 2008, ApJ, 683, L135

Chevalier, R. A., \& Klein, R. I. 1978, ApJ, 219, 994

Chugai, N. N., \& Danziger, I. J. 1994, MNRAS, 268, 173

Couderc, P. 1939, Ann. Astrophys., 2, 271

Docenko, D., \& Sunyaev, R. A. 2010, A\&A, 509, A59

Doty, S. D., \& Leung, C. M. 1994, ApJ, 424, 729
Dwek, E. 1983, ApJ, 274, 175

Dwek, E., A'Hearn, M. F., Becklin, E. E., et al. 1983, ApJ, 274, 168

Emmering, R. T., \& Chevalier, R. A. 1989, ApJ, 338, 388

Ercolano, B., Barlow, M. J., \& Storey, P. J. 2005, MNRAS, 362, 1038

Fabbri, J., Otsuka, M., Barlow, M. J., et al. 2011, MNRAS, 418, 1285

Fesen, R. A., \& Becker, R. H. 1990, ApJ, 351, 437

Fesen, R. A., Gerardy, C. L., Filippenko, A. V., et al. 1999, AJ, 117, 725

Fesen, R. A., Hurford, A. P., \& Matonick, D. M. 1995, AJ, 109, 2608

Fesen, R. A., \& Matonick, D. M. 1994, ApJ, 428, 157

Fesen, R. A., Morse, J. A., Chevalier, R. A., et al. 2001, AJ, 122, 2644

Fesen, R. A., Zastrow, J. A., Hammell, M. C., Shull, J. M., \& Silvia, D. W. 2011, ApJ, 736, 109

France, K., McCray, R., Heng, K., et al. 2010, Science, 329, 1624

Gerardy, C. L., Fesen, R. A., Höflich, P., \& Wheeler, J. C. 2000, AJ, 119, 2968

Gezari, S., Dessart, L., Basa, S., et al. 2008, ApJ, 683, L131

Ghavamian, P., Hughes, J. P., \& Williams, T. B. 2005, ApJ, 635, 365

Groh, J. H., Hillier, D. J., Damineli, A., et al. 2009, ApJ, 698, 1698

Hammer, N. J., Janka, H.-T., \& Muller, E. 2010, ApJ, 714, 1371

Henyey, L. C., \& Greenstein, J. L. 1941, ApJ, 93, 70

Karachentsev, I. D., Sharina, M. E., \& Huchtmeier, W. K. 2000, A\&A, 362, 544

Kennicutt, R. C., Calzetti, D., Aniano, G., et al. 2011, PASP, 123, 1347

Koekemoer, A., Fruchter, A., Hook, R., \& Hack, W. 2002, in HST Calibration Workshop, Hubble after the Installation of the ACS and the NICMOS Cooling System, ed. S. Arribas, A. Koekemoer, \& B. Whitmore (Baltimore, MD: Space Telescope Science Institute), 337

Laor, A., \& Draine, B. T. 1993, ApJ, 402, 441

Leibundgut, B., Kirshner, R. P., Pinto, P. A., et al. 1991, ApJ, 372, 531

Leibundgut, B., Kirshner, R. P., \& Porter, A. C. 1993, BAAS, 25, 834

Lucy, L. B., Danziger, I. J., Gouiffes, C., \& Bouchet, P. 1989, in IAU Colloq. 120, Structure and Dynamics of the Interstellar Medium, ed. G. TenorioTagle, M. Moles, \& J. Melnick (Lecture Notes in Physics, Vol. 350; Berlin: Springer), 164

Makovoz, D., \& Khan, I. 2005, in ASP Conf. Ser. 347, Astronomical Data Analysis Software and Systems XIV, ed. P. Shopbell, M. Britton, \& R. Ebert (San Francisco, CA: ASP), 81

Mathis, J. S., Rumpl, W., \& Nordsieck, K. H. 1977, ApJ, 217, 425

Matsuura, M., Dwek, E., Meixner, M., et al. 2011, Science, 333, 1258

Michael, E., McCray, R., Pun, C. S. J., et al. 2000, ApJ, 542, L53

Monet, D. G., Levine, S. E., Canzian, B., et al. 2003, AJ, 125, 984

Montes, M. J., van Dyk, S. D., Weiler, K. W., Sramek, R. A., \& Panagia, N. 1998, ApJ, 506, 874 
Nakar, E., \& Sari, R. 2010, ApJ, 725, 904

Patat, F. 2005, MNRAS, 357, 1161

Pozzo, M., Meikle, W. P. S., Fassia, A., et al. 2004, MNRAS, 352, 457

Press, W. H., Teukolsky, S. A., Vetterling, W. T., \& Flannery, B. P. 1992, Numerical Recipes in Fortran 77 (2nd ed.; Cambridge: Cambridge Univ. Press)

Pun, C. S. J., Michael, E., Zhekov, S. A., et al. 2002, ApJ, 572, 906

Rabinak, I., \& Waxman, E. 2011, ApJ, 728, 63

Rajagopal, J., Menut, J.-L., Wallace, D., et al. 2007, ApJ, 671, 2017

Rowan-Robinson, M. 1980, ApJS, 44, 403

Rybicky, G. B., \& Lightman, A. P. 1985, Radiative Processes in Astrophysics (New York: Wiley)

Sgro, A. G. 1975, ApJ, 197, 621

Sugerman, B. E. K. 2003, AJ, 126, 1939

Sugerman, B. E. K. 2005, ApJ, 632, L17

Sugerman, B. E. K., \& Crotts, A. P. S. 2002, ApJ, 581, L97

Sugerman, B. E. K., Crotts, A. P. S., Kunkel, W. E., Heathcote, S. R., \& Lawrence, S. S. 2005, ApJS, 159, 60
Sugerman, B. E. K., Lawrence, S. S., Crotts, A. P. S., Bouchet, P., \& Heathcote, S. R. 2002, ApJ, 572, 209

Uomoto, A. 1991, AJ, 101, 1275

Uomoto, A., \& Kirshner, R. P. 1986, ApJ, 308, 685

Városi, F., \& Dwek, E. 1999, ApJ, 523, 265 (VD99)

Voors, R. H. M., Waters, L. B. F. M., de Koter, A., et al. 2000, A\&A, 356, 501

Warren, J. S., Hughes, J. P., \& Slane, P. O. 2003, ApJ, 583, 260

Weiler, K. W., van Dyk, S. D., Panagia, N., \& Sramek, R. A. 1992, ApJ, 398, 248

Weingartner, J. C., \& Draine, B. T. 2001, ApJ, 548, 296 (WD01)

Weis, K. 2011, in IAU Symp. 272, Active OB Stars: Structure, Evolution, Mass Loss, and Critical Limits, ed. C. Neiner, G. Wade, G. Meynet, \& G. Peters (Cambridge: Cambridge Univ. Press), 372

Welch, D. L., Clayton, G. C., Campbell, A., et al. 2007, ApJ, 669, 525

Wild, P., \& Barbon, R. 1980, IAU Circ., 3532, 1

Xu, J., Crotts, A. P. S., \& Kunkel, W. E. 1995, ApJ, 451, 806 (erratum 463, 391) 